\title{
On tails of stationary measures on a class of solvable groups
}

\author{
Dariusz Buraczewski ${ }^{1}$ \\ Institute of Mathematics, Wroclaw University, 50-384 Wroclaw, pl. Grunwaldzki 2/4, Poland \\ Received 13 December 2005; received in revised form 30 May 2006; accepted 4 July 2006 \\ Available online 14 December 2006
}

\begin{abstract}
Let $G$ be a subgroup of GL( $\mathbb{R}, d)$ and let $\left(Q_{n}, M_{n}\right)$ be a sequence of i.i.d. random variables with values in $\mathbb{R}^{d} \rtimes G$ and law $\mu$. Under some natural conditions there exists a unique stationary measure $v$ on $\mathbb{R}^{d}$ of the process $X_{n}=M_{n} X_{n-1}+Q_{n}$. Its tail properties, i.e. behavior of $v\{x:|x|>t\}$ as $t$ tends to infinity, were described some over thirty years ago by H. Kesten, whose results were recently improved by B. de Saporta, Y. Guivarc'h and E. Le Page. In the present paper we study the tail of $v$ in the situation when the group $G_{0}$ is Abelian and $\mathbb{R}^{d}$ is replaced by a more general nilpotent Lie group $N$. Thus the tail behavior of $v$ is described for a class of solvable groups of type $N A$, i.e. being semi-direct extension of a simply connected nilpotent Lie group $N$ by an Abelian group isomorphic to $\mathbb{R}^{d}$. Then, due to A. Raugi, $(N, v)$ can be interpreted as the Poisson boundary of $(N A, \mu)$.
\end{abstract}

(c) 2006 Elsevier Masson SAS. All rights reserved.

\section{Résumé}

Soit $G$ un sous groupe de $\operatorname{GL}(\mathbb{R}, d)$ et soit $\left(Q_{n}, M_{n}\right) \in \mathbb{R}^{d} \rtimes G$ une suite de variables aléatoires indépendantes de loi $\mu$. Sous des hypothèses convenables il y a une unique mesure stationnaire $v$ sur $\mathbb{R}^{d}$ pour le processus auto-régressif linéaire $X_{n}=$ $M_{n} X_{n-1}+Q_{n}$. Les propriétés asymptotiques de la queue $v\{x:|x|>t\}, t \rightarrow \infty$, ont été étudiées par $\mathrm{H}$. Kesten il y a 30 ans et plus récemment de nouveaux résultats ont été obtenus par B. de Saporta, Y. Guivarc'h and E. Le Page. Dans cet article on étudie le cas où $G$ est abélien et $\mathbb{R}^{d}$ est remplacé par un groupe de Lie nilpotent $N$. On obtient alors le comportement à l'infini de la queue de $v$ pour une classe particulière de groupes de tyle $N A$ produits semi-direct d'un groupe $N$ simplement connexe $N$ avec $G=\mathbb{R}^{d}$. Dans ce cas pariculier $(N, v)$ est un bord de Poisson au sens de A. Raugi.

(c) 2006 Elsevier Masson SAS. All rights reserved.

Keywords: Solvable Lie groups; Stationary measure; Poisson kernel

\section{Introduction}

We study random recursions on solvable Lie groups $S$, which satisfy the following assumptions

- $S$ is the semi-direct product of an Abelian group $A$, isomorphic to $\mathbb{R}^{d}$, acting on a simply connected nilpotent Lie group $N$,

E-mail address: dbura@math.uni.wroc.pl (D. Buraczewski).

1 Research partially supported by KBN grant 1 P03A 018 26. The manuscript was prepared when the author was staying at Department of Mathematics, Université de Rennes and at Department of Mathematics, University Pierre \& Marie Curie, Paris VI. The visits were financed by the European Commission IHP Network 2002-2006 Harmonic Analysis and Related Problems (Contract Number: HPRN-CT-2001-00273 - HARP) and European Commission Marie Curie Host Fellowship for the Transfer of Knowledge "Harmonic Analysis, Nonlinear Analysis and Probability", MTKD-CT-2004-013389. The author would like to express his gratitude to the hosts for hospitality. 
- there exists a contracting element $a_{0} \in A$, i.e. for every $x \in N: \lim _{k \rightarrow \infty} \delta_{a_{0}}^{k}(x)=0$, where $\delta_{a_{0}}$ stands for the action of $a_{0}$ on $N$, and 0 is the unit element in $N$.

Various classical objects like symmetric spaces, bounded homogeneous domains in $\mathbb{C}^{n}$ and manifolds of negative curvature admit simply transitive actions of such groups and therefore they are of considerable interest from many points of view $[1,16,20]$.

Given a probability measure $\mu$ on $S$ we define a random walk

$$
S_{n}=X_{n} \cdots X_{1},
$$

where $\left\{X_{i}\right\}_{i=1}^{\infty}$ is a sequence of independent identically distributed (i.i.d.) random variables with law $\mu$.

We write

$$
X_{1}=Q M,
$$

with $Q=\pi_{N}\left(X_{1}\right) \in N, M=\pi_{A}\left(X_{1}\right) \in A$, where $\pi_{N}$ and $\pi_{A}$ denote canonical projections of $S$ onto $N$ and $A$, respectively. We shall assume that

- $\mu$ is mean-contracting, that is the element of the group corresponding to the vector $\int_{S} \log M \mathrm{~d} \mu(Q, M)$ is contracting;

- $\int_{S}\left(|\log \|M\||+\log ^{+}|Q|\right) \mathrm{d} \mu(Q, M)<\infty$, for convenient norms on $A$ and $N$ that will be defined in Section 2.

Under these hypotheses the limit $R$ of $\pi_{N}\left(S_{n}\right)$ exists in law (A. Raugi [21]) and gives rise to the measure $v$ that is the only stationary measure for the Markov chain $\pi_{N}\left(S_{n}\right)$ i.e.

$$
\mu * v=v .
$$

This means that for every positive, Borel measurable function $f$ on $N$, we have

$$
\mu * v(f)=\int f\left(\pi_{N}(g \cdot x)\right) \mu(\mathrm{d} g) v(\mathrm{~d} x)=v(f) .
$$

Moreover, if $\mu$ is spread out (i.e. some power of $\mu$ is nonsingular with respect to the Haar measure on $S$ ) and its support generates the group $S$, A. Raugi [21] proved that $(N, v)$ is the Poisson boundary of this process, i.e. using the stationary measure $v$ one can reconstruct bounded $\mu$-harmonic functions on $S$, knowing their boundary value on $N$.

Our aim is to study behavior of

$$
v\{x:|x|>t\}=\mathbb{P}[|R|>t]
$$

as $t$ tends to infinity, provided some further hypothesis on $\mu$.

When the Abelian group is one dimensional, i.e. $A=\mathbb{R}^{+}$, the tail behavior is well understood. If $N=\mathbb{R}$, it was observed by H. Kesten [17] that the tail behavior of $v$ is strictly related to properties of the Laplace transform of $\pi_{A}(\mu)$ and that under natural conditions there exists $\alpha>0$ such that

$$
\lim _{t \rightarrow \infty} t^{\alpha} \mathbb{P}[|R|>t]=C,
$$

for some positive constant $C$. His proof was later essentially simplified by A.K. Grincevičius [12] and Ch. Goldie [11]. The general situation of solvable groups being extensions of nilpotent groups by one-dimensional Abelian group of automorphisms was studied in [2], where similar results were obtained. Much more can be said about $v$ when the measure $\mu$ comes from a second-order, subelliptic, left-invariant differential operator $\mathcal{L}$ on $S$, i.e. when instead of $\mu$ we consider a semigroup of measures $\mu_{t}$, whose infinitesimal generator is $\mathcal{L}$, and the measure $v$ satisfies

$$
\breve{\mu}_{t} * v=v \quad \text { for every } t \text {. }
$$

Then, the measure $v$ has a density and its behavior along some rays tending to infinity has been described in [6] and [3].

The situation when the group $A$ acting on $N$ is multidimensional is much more complicated. In the context of general solvable groups, the only results we know, concerning behavior at infinity of the stationary measure, were obtained in some particular cases when the measure $\mu$ is connected with an subelliptic operator on $S$ (compare above). 
If $X=G / K$ is a noncompact symmetric space, $S$ is the solvable part of the Iwasawa decomposition of $G=S K$ and $\mathcal{L}$ is the Laplace-Beltrami operator, $v$ has a smooth density $m$, called Poisson kernel, which can be explicitly computed (see e.g. [8]). The formulas however are not very transparent as far as the pointwise decay at $\infty$ is concerned.

More general situation was studied by E. Damek and A. Hulanicki [4,5]. They considered on solvable groups $S=N A$, with diagonal action of $A$ on $N$, a large class of left-invariant second order, degenerate elliptic operators $\mathcal{L}$ and identified the Poisson boundary of $(S, \mathcal{L})$ with $\left(N_{1}, v\right)$, where $N_{1}$ is some normal subgroup of $N$. Then the stationary measure $v$ on $N_{1}$ has again smooth density $m$ and they proved, without knowing an explicit formula for $m$ that

$$
\int_{N_{1}} \tau_{N_{1}}(x)^{\varepsilon} m(x) \mathrm{d} x<\infty,
$$

for some positive $\varepsilon$, where $\tau_{N_{1}}$ is the Riemannian distance of $x$ from the identity, and $d x$ is the Haar measure on $N$.

The case when $N$ is an Euclidean space, but the measure is general (not coming from a differential operator) was studied by many authors. Assume $N=\mathbb{R}^{m}$ and there exists a group of matrices $G$ (not necessarily Abelian) acting on $\mathbb{R}^{m}$. Consider the stochastic recursion

$$
R_{n+1}=M_{n+1} R_{n}+Q_{n+1},
$$

where $\left(Q_{n}, M_{n}\right)$ is a sequence of i.i.d., $\mathbb{R}^{m} \times G$ valued random variables distributed according to the given probability measure $\mu$. Then under suitable assumptions $R_{n}$ converges to a random variable $R$, whose distribution $v$ is $\mu$-invariant. Asymptotic properties of $R$ were studied by several authors [17-19,7,14]. Their main assumptions (except meancontractivity and finiteness of some exponential moments) were proximality and (or) irreducibility. Let $\bar{\mu}$ be the canonical projection of $\mu$ onto $G$. Then proximality means that the semigroup generated by the support of $\bar{\mu}$ contains a proximal element, i.e. a matrix having a unique real dominant eigenvalue (i.e. the corresponding eigenspace is onedimensional). The action is called irreducible if there does not exist a finite union of proper subspaces of $\mathbb{R}^{m}$, which is invariant under the action of the support of $\bar{\mu}$.

In this paper we study the reducible situation on general solvable groups. Our assumptions are natural generalization of one-dimensional situation, i.e. first of all we require finiteness of some exponential moments of $\pi_{A}(\mu)$. The main results of the paper are presented in Section 3.4 as Main Theorem A and Main Theorem B. In full generality we prove that there exists a constant $\chi_{0}$ such that for any $\varepsilon>0$

$$
C_{1} t^{-\chi_{0}} \leqslant \mathbb{P}[|R|>t] \leqslant C_{\varepsilon} t^{-\left(\chi_{0}-\varepsilon\right)},
$$

where $C_{1}$ and $C_{\varepsilon}$ are positive constants, and $C_{\varepsilon}$ depends on $\varepsilon$. Notice that the result is new even in the case when an Abelian group of matrices $A=G$ acts on $N=\mathbb{R}^{m}$ and the measure $\mu$ does not satisfies to the assumptions of proximality and irreducibility required by the papers mentioned above.

We obtain more detailed description of the tail of the measure $\nu$, when the action of $A$ is fully reducible, i.e. $A$ acts diagonally on $N$. This corresponds to the classical situations of symmetric spaces and bounded homogeneous domains. Then we prove, without assuming proximality of $\mu$, the existence of positive constants $\chi_{0}$ and $C_{2}$ such that

$$
C_{1} t^{-\chi_{0}} \leqslant \mathbb{P}[|R|>t] \leqslant C_{2} t^{-\chi_{0}}
$$

If we assume existence of a dominant root (see Section 3 for precise definitions), that in some sense substitutes the notion of proximality, we show

$$
\lim _{t \rightarrow \infty} t^{\chi_{0}} \mathbb{P}[|R|>t]=C_{3},
$$

for some $C_{3}>0$.

The outline of the paper is as follows. In Section 2 we introduce a class of solvable Lie groups for which our results holds and describe precisely their structure. In Section 3 we include a brief account of random walks on solvable groups: existence of an invariant measure and its properties in the case when the group $A$ is one-dimensional. Then we describe our assumptions and state the main results of the article. Their proofs are contained in Sections 4 and 5 , respectively.

The author is grateful to the referee for helpful comments and corrections, improving the presentation of this paper and some arguments in the proof. 


\section{A class of solvable Lie groups}

Let $A$ be an Abelian group isomorphic to $\mathbb{R}^{d}$, acting on a nilpotent, connected and simply connected Lie group $N$, i.e.

$$
\delta_{a}(x y)=\delta_{a}(x) \delta_{a}(y), \quad a \in A, x, y \in N,
$$

where $\delta_{a}$ denotes the action of $a \in A$ on $N$.

The semi-direct product $N \rtimes A$ is a solvable Lie group denoted by $S$. We shall denote by $\circ$ the action of the group $S$ on $N$, i.e.

$$
(x, a) \circ y=x \cdot \delta_{a}(y), \quad \text { for }(x, a) \in S \text { and } y \in N .
$$

Then the group multiplication in $S$ is given by

$$
(x, a) \cdot(y, b)=((x, a) \circ y, a b) .
$$

Let $e(0, I$ respectively) be the neutral element of $S$ ( $N, A$ respectively).

Our main assumption on $S$ is that the action of $A$ on $N$ is contractive i.e. that there exists an element $a \in A$ such that

$$
\lim _{k \rightarrow \infty} \delta_{a}^{k}(x)=0, \quad \text { for every } x \in N .
$$

The Lie algebras of $A, N, S$ are denoted by $\mathcal{A}, \mathcal{N}$ and $\mathcal{S}$ respectively. Then $\mathcal{S}=\mathcal{N} \oplus \mathcal{A}$ and of course for every $H \in \mathcal{A}$, ad $H$ preserves $\mathcal{N}$. The exponential maps are global diffeomorphisms both between $\mathcal{N}$ and $N$, and between $\mathcal{A}$ and $A$. Their inverse will be denoted by $\log$. Then for any $X \in \mathcal{N}$

$$
\delta_{a}(\exp (X))=\exp \left(\mathrm{e}^{\mathrm{ad}(\log a)} X\right) .
$$

We shall denote the foregoing action of the group $A$ on the Lie algebra $\mathcal{N}$, using the same symbol $\delta_{a}(X)$. Let $\mathcal{N}^{\mathbb{C}}$ $\left(N^{\mathbb{C}}\right)$ be the complexification of $\mathcal{N}\left(N\right.$ respectively). For any $\lambda$ in the set $\left(\mathcal{A}^{*}\right)^{\mathbb{C}}$ of continuous homomorphisms from $\mathcal{A}$ to $(\mathbb{C},+)$ define

$$
\mathcal{N}_{\lambda}^{\mathbb{C}}=\left\{Z \in \mathcal{N}^{\mathbb{C}}: \text { there exists } k \text { such that }(\operatorname{ad} H-\lambda I)^{k} Z=0 \text {, for any } H \in \mathcal{A}\right\} .
$$

Then, it is known that for $\lambda_{1}, \lambda_{2} \in\left(\mathcal{A}^{*}\right)^{\mathbb{C}}$

$$
\left[\mathcal{N}_{\lambda_{1}}^{\mathbb{C}}, \mathcal{N}_{\lambda_{2}}^{\mathbb{C}}\right] \subset \mathcal{N}_{\lambda_{1}+\lambda_{2}}^{\mathbb{C}} \text {. }
$$

Moreover any space $\mathcal{N}_{\lambda}^{\mathbb{C}}$ is preserved by the action of the group $A$, i.e.

$$
\delta_{a}(Z) \in \mathcal{N}_{\lambda}^{\mathbb{C}}, \quad \text { for } Z \in \mathcal{N}_{\lambda}^{\mathbb{C}} .
$$

We shall say that $\lambda$ is a root if the appropriate space $\mathcal{N}_{\lambda}^{\mathbb{C}}$ is nonempty. The set of all roots will be denoted by $\Delta$. Then, of course, if $\lambda \in \Delta$ then also $\bar{\lambda} \in \Delta$ and

$$
\mathcal{N}^{\mathbb{C}}=\bigoplus_{\lambda \in \Delta} \mathcal{N}_{\lambda}^{\mathbb{C}}
$$

Let $i_{\lambda}=\operatorname{dim}_{\mathbb{C}} \mathcal{N}_{\lambda}^{\mathbb{C}}$. For any $\lambda$ choose a basis $\left\{Z_{\lambda, 1}, \ldots, Z_{\lambda, i_{\lambda}}\right\}$ of $\mathcal{N}_{\lambda}^{\mathbb{C}}$, such that with respect to this basis $\mathcal{A}$ acts triangularly, i.e. for any $H \in \mathcal{A}$

$$
\text { ad } H\left(Z_{\lambda, j}\right)=\lambda(H) Z_{\lambda, j}+W_{\lambda, j-1},
$$

for some $W_{\lambda, j-1} \in \operatorname{span}\left\{Z_{\lambda, 1}, \ldots, Z_{\lambda, j-1}\right\}$. Then $i_{\lambda}=i_{\bar{\lambda}}$, moreover may assume that $\overline{Z_{\lambda, j}}=Z_{\bar{\lambda}, j}$ and if $\lambda$ is real then all the vectors $Z_{\lambda, j}$ are real.

For a chosen basis $\left\{H_{1}, \ldots, H_{d}\right\}$ of $\mathcal{A}$ introduce coordinates in $A$ : any element $H$ of $\mathcal{A}$ can be uniquely written as $H=\sum t_{i}(H) H_{i}$. Notice that one can compute the action of $A$ on $\mathcal{N}^{\mathbb{C}}$, taking (2.3) and (2.7) into account, we obtain

$$
\delta_{\exp H}\left(Z_{\lambda, k}\right)=\mathrm{e}^{\lambda(H)} \cdot \sum_{j \leqslant k} P_{\lambda, k, j}(H) Z_{\lambda, j},
$$


where $P_{\lambda, k, k}=1$, and $P_{\lambda, k, j}$ for $j$ smaller that $k$ are some polynomials of $t_{i}(H)$. One can easily see that the polynomials depend on $t_{i}(H)$ only if $\lambda\left(H_{i}\right) \neq 0$.

Thus, the assumption that the action of $A$ is contractive implies that the negative Weyl chamber

$$
\mathcal{A}^{--}=\{H \in \mathcal{A}: \mathfrak{R} \lambda(H)<0 \text { for all } \lambda \in \Delta\}
$$

is not empty. Let $\mathcal{A}^{++}=-\mathcal{A}^{--}$be the positive Weyl chamber.

For any $z \in N^{\mathbb{C}}$ let $z_{\lambda, i}$ denotes its $\lambda, i$ component, i.e.

$$
z=\exp \left(\sum z_{\lambda, i} Z_{\lambda, i}\right) \text {. }
$$

A root $\lambda_{0}$ will be called simple if it cannot be written as a sum of other roots, i.e. for all possible choices of nonnegative integer numbers $\left\{c_{\lambda}\right\}_{\lambda \in \Delta}$, such that $\sum c_{\lambda}>1$,

$$
\lambda_{0} \neq \sum_{\lambda \in \Delta} c_{\lambda} \lambda
$$

The set of all simple roots will be denoted by $\Delta_{1}$.

For instance, let $A=\mathbb{R}^{2}$, choose two vector fields $H_{1}, H_{2}$ forming a basis of $\mathcal{A}$, and denote by $\lambda_{1}, \lambda_{2}$ two functionals on $\mathcal{A}$ such that $\lambda_{i}\left(H_{j}\right)=\delta_{i j}$. Then, if $\Delta=\left\{\lambda_{1}, \lambda_{1} / 2,\left(\lambda_{1}+\lambda_{2}\right) / 2, \lambda_{1}+2 \lambda_{2}, \lambda_{2}\right\}$, the set of simple roots consists of three elements: $\Delta_{1}=\left\{\lambda_{1} / 2,\left(\lambda_{1}+\lambda_{2}\right) / 2, \lambda_{2}\right\}$.

We have the following simple lemma

Lemma 2.10. Any root $\lambda_{0}$ can be written in the form

$$
\lambda_{0}=\sum_{\lambda \in \Delta_{1}} c_{\lambda} \lambda
$$

where $c_{\lambda}$ are nonnegative integer numbers.

Proof. Suppose $H \in \mathcal{A}^{++}$and let us number all the roots $\lambda_{1}, \lambda_{2}, \ldots, \lambda_{k}$ in the following way

$$
\mathfrak{R} \lambda_{1}(H) \leqslant \mathfrak{R} \lambda_{2}(H) \leqslant \cdots \leqslant \Re \lambda_{k}(H) .
$$

We shall proceed by induction. Of course, $\lambda_{1}$ is a simple root and (2.11) holds with $c_{\lambda_{1}}=1$. Assume the lemma holds for $\lambda_{1}, \ldots, \lambda_{i-1}$. If the root $\lambda_{i}$ is simple then it satisfies (2.11). Otherwise, $\lambda_{i}$ can be written as

$$
\lambda_{i}=\sum_{\lambda \in \Delta} c_{\lambda} \lambda
$$

where $c_{\lambda}$ are positive integer and $\sum c_{\lambda}>1$. Therefore $\mathfrak{R} \lambda_{i}(H)>\mathfrak{R} \lambda(H)$ for any $\lambda$ such that $c_{\lambda}$ is nonzero. But this set contains either simple roots or other roots satisfying already (2.11). Therefore (2.11) also holds for $\lambda_{i}$.

The group multiplication in $N$ is given by the Campbell-Hausdorf formula:

$$
\exp (X) \cdot \exp (Y)=\exp (X+Y+[X, Y] / 2+\cdots), \quad \text { for } X, Y \in \mathcal{N} .
$$

Since the Lie algebra $\mathcal{N}$ is nilpotent, the sum above is finite. In particular if we fix a simple root $\lambda_{0}$, then in view of (2.5)

$$
(x \cdot y)_{\lambda_{0}, i}=x_{\lambda_{0}, i}+y_{\lambda_{0}, i},
$$

for $x, y \in N$ and $i \leqslant i_{\lambda_{0}}$. We shall describe the Campbell-Hausdorf formula more precisely later in Section 5.

\subsection{Norms on $N$ and $A$}

Now we are going to construct a norm on $N$ adapted to the action of $A$. In the case $A$ is one-dimensional and diagonalizable W. Hebisch and A. Sikora [15] have built on $N$ a smooth outside zero norm, homogeneous on the action of one-dimensional group of dilations, i.e. satisfying $\left|\delta_{a}(x)\right|=a|x|$. Their ideas were used later in [2] to 
construct a homogeneous norm with respect to general one-dimensional group of dilations $A$. Here we shall adopt the construction for our purpose. Since we will need some further properties of the norm we give some details.

Fix $H_{0} \in \mathcal{A}^{++}$such that $\mathfrak{R} \lambda\left(H_{0}\right)>1$ for all roots $\lambda$ and let $A_{0}=\left\{\exp t H_{0}, t \in \mathbb{R}\right\}$ be an one parameter subgroup of $A$. We change coordinates in $A_{0}$, identifying

$$
A_{0} \ni \exp t H_{0} \sim \mathrm{e}^{t} \in \mathbb{R}^{+} .
$$

For $b \in \mathbb{R}^{+}$and $z \in N^{\mathbb{C}}$ define

$$
\sigma_{b}(x)=\delta_{\exp (\log b) H_{0}}(z) .
$$

Then $\sigma$ defines the action of $\mathbb{R}^{+}$on $N^{\mathbb{C}}$, preserving $N$, and the semi-direct product $N \rtimes \mathbb{R}^{+}$is a solvable group, belonging to the class of solvable Lie groups studied in [2]. A key step of the construction is the following lemma:

Lemma 2.15. ([15,2]) There exists an open rectangle

$$
\Omega=\left\{Z=\sum_{\lambda, i} z_{\lambda, i} Z_{\lambda, i} \in \mathcal{N}^{\mathbb{C}}:\left|z_{\lambda, i}\right|<c_{\lambda, i}\right\},
$$

where $c_{\lambda, i}$ are some positive constants, such that

$$
\text { if } \log (z), \log (w) \in \Omega, \text { for } z, w \in N^{\mathbb{C}} \text { and } 0<b<1 \text { then } \log \left(\sigma_{b}(z) \sigma_{1-b}(w)\right) \in \Omega \text {. }
$$

We define the norm on $N^{\mathbb{C}}$ :

$$
|z|=\inf \left\{b: \log \left(\sigma_{b^{-1}}(z)\right) \in \Omega\right\}=\inf \left\{\mathrm{e}^{t}: \log \left(\delta_{\exp t H_{0}}^{-1}(z)\right) \in \Omega\right\} .
$$

One can easily check that this norm is continuous and satisfies to the following properties

- $|\cdot|$ is symmetric: $\left|z^{-1}\right|=|z|$;

- $|z|=0$ if and only if $z=0$;

- $|\cdot|$ is subadditive, i.e. $|z \cdot w| \leqslant|z|+|w|$;

- $\left|\sigma_{b}(z)\right|=b|z|$, for any $b \in \mathbb{R}^{+}$.

Finally, we define a norm on $A$ :

$$
\|a\|=\max _{|z|=1}\left|\delta_{a}(z)\right| .
$$

Observe that

$$
\left|\delta_{a}(z)\right| \leqslant\|a\||z| \text { and } \quad\left\|a_{1} a_{2}\right\| \leqslant\left\|a_{1}\right\|\left\|a_{2}\right\| .
$$

We shall often use the following constants being closely related to properties of the foregoing norms

$$
d_{\lambda}=\mathfrak{R} \lambda\left(H_{0}\right), \quad \lambda \in \Delta,
$$

and their simple property

$$
\text { if } \lambda_{0}=\sum c_{\lambda} \lambda \text {, then } d_{\lambda_{0}}=\sum c_{\lambda} d_{\lambda} .
$$

A crucial step in the proof of our main results will be the following lemma:

Lemma 2.19. There exist constants $C$ and $D$ such that

$$
\|a\| \leqslant C \max _{\lambda \in \Delta}\left\{\mathrm{e}^{\frac{\Re \lambda(H)}{d_{\lambda}}}\right\} \cdot\left(1 \vee \max _{i}\left|t_{i}(H)\right|^{D}\right)
$$

for any $a=\exp H \in A$, where $t_{i}(H)$ denotes ith coordinate of $H$ in the fixed basis of $\mathcal{A}$. 
Proof. First, we shall prove that

$$
\|a\| \leqslant \max _{\lambda \in \Delta} \sup _{\left\{z_{\lambda} \in N_{\lambda}^{\mathbb{C}}:\left|z_{\lambda}\right|=1\right\}}\left|\delta_{a}\left(z_{\lambda}\right)\right| .
$$

In fact, every space $\mathcal{N}_{\lambda}^{\mathbb{C}}$ is invariant under the action of $A(2.6)$, and writing any element of $N^{\mathbb{C}}$ as $z=$ $\exp \left(\sum_{\lambda, i} z_{\lambda, i} Z_{\lambda, i}\right)$ and using the fact that the action of $A$ on $\mathcal{N}^{\mathbb{C}}$ is linear we have

$$
\begin{aligned}
\|a\| & =\sup _{|z|=1}\left|\delta_{a}(z)\right|=\sup _{|z|=1} \inf \left\{b: \sigma_{b^{-1}} \delta_{a}\left(\sum_{\lambda, i} z_{\lambda, i} Z_{\lambda, i}\right) \in \Omega\right\} \\
& =\sup _{|z|=1} \inf \left\{b: \sigma_{b^{-1}} \delta_{a}\left(\sum_{i} z_{\lambda, i} Z_{\lambda, i}\right) \in \Omega \text { for all roots } \lambda\right\} \\
& \leqslant \max _{\lambda \in \Delta} \sup _{\left\{z_{\lambda} \in N_{\lambda}^{\mathbb{C}}:\left|z_{\lambda}\right|=1\right\}}\left|\delta_{a}\left(z_{\lambda}\right)\right|,
\end{aligned}
$$

which proves desired inequality (2.20).

Define the function $g(H)=\max _{i}\left|t_{i}(H)\right|$. In view of (2.20) it is enough to justify that for any root $\lambda$ there exist constants $C_{\lambda}, D_{\lambda}$ such that if $g(H)>C_{\lambda}$, then

$$
\sigma_{b}^{-1} \delta_{\exp H}\left(Z_{\lambda}\right) \in \Omega
$$

for $b=\exp \left\{\frac{\Re \lambda(H)}{d_{\lambda}}\right\} \cdot\left(1 \vee g(H)^{D_{\lambda}}\right)$ and any $Z_{\lambda}=\sum_{i} z_{\lambda, i} Z_{\lambda, i} \in \bar{\Omega} \cap \mathcal{N}_{\lambda}^{\mathbb{C}}$. In view of (2.8)

$$
\delta_{a}\left(Z_{\lambda}\right)=\mathrm{e}^{\lambda(H)} \cdot \sum_{k} z_{\lambda, k}\left(\sum_{j \leqslant k} P_{\lambda, k, j}(H) Z_{\lambda, j}\right),
$$

where $P_{\lambda, k, j}$ are some polynomials of $t_{j}(H)$ and $P_{\lambda, k, k}=1$.

Next we have

$$
\begin{aligned}
\sigma_{b}^{-1} \delta_{a}\left(Z_{\lambda}\right) & =\delta_{\exp (-\log b) H_{0} \delta_{a}\left(Z_{\lambda}\right)} \\
& =\mathrm{e}^{-\log b \cdot \lambda\left(H_{0}\right)+\lambda(H)} \cdot \sum_{k} z_{\lambda, k}\left(\sum_{j} \bar{P}_{\lambda, k, j}(H, \log b) Z_{\lambda, j}\right),
\end{aligned}
$$

where $\bar{P}_{\lambda, k, j}$ are some polynomials of $t_{j}(H)$ and $\log b$. Substituting $b$ in the formula above we obtain

$$
\begin{aligned}
\sigma_{b}^{-1} \delta_{a}\left(Z_{\lambda}\right)= & (1 \vee g(H))^{-D_{\lambda} \Re \lambda\left(H_{0}\right)} \cdot \mathrm{e}^{-\mathrm{i} \mathfrak{I} \lambda\left(H_{0}\right)\left(\frac{\mathfrak{\Re} \lambda(H)}{\mathfrak{R} \lambda\left(H_{0}\right)}+D_{\lambda} \log ^{+} g(H)\right)} \cdot \mathrm{e}^{\mathrm{i} \Im \lambda(H)} \\
& \times \sum_{k}\left(\sum_{j} \overline{\bar{P}}_{\lambda, k, j}\left(t_{i}(H), D_{\lambda} \log ^{+} g(H), \Re \lambda(H)\right) z_{\lambda, j}\right) Z_{\lambda, k},
\end{aligned}
$$

where $\overline{\bar{P}}_{\lambda, k, j}$ are polynomials coming from appropriately modified polynomials $\bar{P}_{\lambda, k, j}$ and degrees of these polynomials depends only on the structure of the solvable group $S$. Finally, choosing $D_{\lambda}$ large enough, there exists $C_{\lambda}$ such that if $g(H)>C_{\lambda}$ then for all $k$

$$
(1 \vee g(H))^{-D_{\lambda} \Re \lambda\left(H_{0}\right)} \sum_{j}\left|\overline{\bar{P}} P_{\lambda, k, j}\left(t_{i}(H), D_{\lambda} \log ^{+} g(H), \Re \lambda(H)\right)\right|\left|z_{\lambda, j}\right| \leqslant c_{\lambda, k},
$$

which proves the lemma.

\section{Random walks on $N A$ groups and main theorems}

\subsection{Random walks}

Given a probability measure $\mu$ on $S$ we define a random walk:

$$
S_{n}=\left(Q_{n}, M_{n}\right) \cdots\left(Q_{1}, M_{1}\right),
$$


where $\left(Q_{n}, M_{n}\right)$ is a sequence of i.i.d. $S$-valued random variables with a distribution $\mu$. The law of $S_{n}$ is the $n$ thconvolution $\mu^{* n}$ of $\mu$.

Our aim is to study the $N$-component of $S_{n}$, i.e. the Markov chain on $N$ generated by the random walk on $S$ :

$$
\begin{aligned}
& R_{n}=\pi_{N}\left(S_{n}\right)=\left(Q_{n}, M_{n}\right) \circ R_{n-1}, \\
& R_{0}=\delta_{0},
\end{aligned}
$$

where $\pi_{N}$ denotes the canonical projection $\pi_{N}: S \rightarrow S / A$. By $\pi_{A}$ we shall denote the analogous projection of $S$ onto $A$.

It was proved by A. Raugi [21] that when $\mu$ is mean-contracting, i.e.

$$
\mathbb{E} \log M=\int \log M \mu_{A}(\mathrm{~d} M) \in \mathcal{A}^{--},
$$

where $\mu_{A}=\pi_{A}(\mu)$, and under the following integrability condition

$$
\mathbb{E}\left|\log \|M\|+\log ^{+}\right| Q||<\infty
$$

(the norms used by A. Raugi were different, but his proof gives the result also in our case) $R_{n}$ converges in law to a random variable $R$, whose distribution will be denoted by $v$, and $R$ does not depend on the choice of $R_{0}$. Moreover, $v$ is a unique stationary solution of the stochastic equation

$$
v=\mu * v,
$$

where

$$
\mu * v(f)=\int f(g \circ x) \mu(\mathrm{d} g) v(\mathrm{~d} x) .
$$

The above equation can be also written in the form

$$
R={ }_{d}(Q, M) \circ R,
$$

where $R$ and $(Q, M)$ are independent distributed according to $v$ and $\mu$, respectively.

The random variable $R$ is constructed as a pointwise limit of the "backward" process:

$$
\begin{aligned}
& R_{0}^{*}=0, \\
& R_{n}^{*}=\pi_{N}\left(\left(Q_{1}, M_{1}\right) \cdots\left(Q_{n}, M_{n}\right)\right)=Q_{1} \cdot \delta_{\Pi_{1}}\left(Q_{2}\right) \cdots \delta_{\Pi_{n-1}}\left(Q_{n}\right),
\end{aligned}
$$

where $\Pi_{n}=M_{1} \cdots M_{n}$.

Our aim is to study, under some additional hypothesis, behavior of

$$
v\{x:|x|>t\}=\mathbb{P}[|R|>t]
$$

as $t$ tends to infinity.

\subsection{Asymptotic behavior of $R$ when $\operatorname{dim} A=1$}

When the Abelian group $A$ is one-dimensional, the behavior of the above sequence is well-known. The simplest example of a solvable group is the "ax $+\mathrm{b}$ " group, i.e. semi-direct product of $N=\mathbb{R}$ and $A=\mathbb{R}^{+}$, with the group action

$$
(x, a) \cdot(y, b)=(x+a y, a b), \quad x, y \in \mathbb{R}, a, b \in \mathbb{R}^{+} .
$$

Then, Kesten [17] proved (under some further assumptions) that there exist positive constants $\alpha$ and $C$ such that

$$
\lim _{t \rightarrow \infty} t^{\alpha} \mathbb{P}[|R|>t]=C .
$$

His proof was later essentially simplified by Grincevičius [12] and Goldie [11]. Their ideas were used in [2] to handle with general situation of homogeneous groups, when the group $S$ is a semi-direct product of a nilpotent group $N$ and of an one-dimensional group of dilations $A=\mathbb{R}^{+}$. In this case the norm $|\cdot|$ is homogeneous for the action of $\mathbb{R}^{+}$, i.e. $\left|\delta_{a}(x)\right|=a|x|$ for every $a \in \mathbb{R}^{+}, x \in N$, and we have the following theorem: 
Theorem 3.4. ([2]) Let $S=N \rtimes \mathbb{R}^{+}$and assume that

- $\mathbb{E} \log M<0$,

- there exists $\alpha>0$, such that $\mathbb{E} M^{\alpha}=1$,

- the law of $\lambda \log M$ is non-arithmetic, i.e. there does not exist $a>0$ such that $\lambda \log M \in a \mathbb{Z}$,

- $\mathbb{E} M^{\alpha} \log M<\infty$,

- $\mathbb{E}|Q|^{\alpha}<\infty$.

Then

$$
\lim _{t \rightarrow \infty} t^{\alpha} \mathbb{P}[|R|>t]=C .
$$

for some constant $C$. Moreover, if the action of $\mathbb{R}^{+}$on $N$ is diagonalizable then the constant $C$ is nonzero if and only iffor every $x \in N$,

$$
\mathbb{P}[(Q, M) \circ x=x]<1 .
$$

If the action is not diagonalizable, the constant $C$ is positive under the additional hypothesis that $|Q|$ is bounded almost surely.

We shall often use description of asymptotic behavior of

$$
\mathbb{P}\left[\max _{n}\left\{M_{1} \cdots M_{n}\right\}>t\right]
$$

where $M_{i}$ are i.i.d. real valued random variables satisfying the assumptions of Theorem 3.4. It was observed by Kesten, that the sequence is strictly connected with asymptotic behavior of $R$. Then it is well known that there exists a positive constant $C$ such that

$$
\lim _{t \rightarrow \infty} t^{\alpha} \mathbb{P}\left[\max _{n}\left\{M_{1} \cdots M_{n}\right\}>t\right]=C
$$

(see [9] for more details).

\subsection{Laplace transform}

In order to describe the tail of $R$ we shall need some further assumptions on $\mu$. Consider the Laplace transform of the measure $\mu_{A}=\pi_{A}(\mu)$ :

$$
L(\alpha)=\int_{A} \mathrm{e}^{\alpha(\log M)} \mu_{A}(\mathrm{~d} M)=\mathbb{E}\left[\mathrm{e}^{\alpha(\log M)}\right]
$$

where $\alpha \in \mathcal{A}^{*}$. We assume that

$$
\text { for any } \lambda \in \Delta \text { there exists } \chi_{\lambda}>0 \text { such that } L\left(\frac{\chi_{\lambda} \Re \lambda}{d_{\lambda}}\right)=\mathbb{E}\left[\mathrm{e}^{\frac{\chi_{\lambda} \Re \lambda(\log M)}{d_{\lambda}}}\right]=1 \text {. }
$$

Then it is known that the Laplace transform is well defined for all functionals on $A$ belonging to the convex hull $V$ of 0 and $\chi_{\lambda} \Re \lambda / d_{\lambda}$ for all roots $\lambda \in \Delta$. Furthermore $L$ is convex on $V$ and because of (3.2) and (3.7) it is strictly smaller than 1 on the set

$$
V_{0}=\left\{\alpha \in \mathcal{A}^{*}: \alpha=\sum_{\lambda \in \Delta} c_{\lambda} \cdot \frac{\chi_{\lambda} \Re \lambda}{d_{\lambda}}, \text { for nonnegative numbers } c_{\lambda} \text { satisfying } 0<\sum c_{\lambda}<1\right\},
$$

i.e.

$$
\text { if } \alpha \in V_{0} \text { then } L(\alpha)<1 \text {. }
$$

Define

$$
\chi_{0}=\min _{\lambda \in \Delta}\left\{\chi_{\lambda}\right\}
$$

then the following holds 
Lemma 3.9. Let $\lambda_{0}=\sum c_{\lambda} \lambda$ for some nonnegative numbers $c_{\lambda}$. Assume that for some root $\lambda_{1}$ the constant $c_{\lambda_{1}}$ is nonzero and $\chi_{\lambda_{1}}>\chi_{0}$. Then $\chi_{\lambda_{0}}>\chi_{0}$.

Proof. Let us write

$$
\frac{\chi_{0} \Re \lambda_{0}}{d_{\lambda_{0}}}=\sum_{\lambda} \frac{\chi_{0} d_{\lambda} c_{\lambda}}{d_{\lambda_{0}} \chi_{\lambda}} \cdot \frac{\chi_{\lambda} \Re \lambda}{d_{\lambda}},
$$

and notice that because of our assumptions and (2.18) we have

$$
\sum_{\lambda} \frac{\chi_{0} d_{\lambda} c_{\lambda}}{d_{\lambda_{0}} \chi_{\lambda}}<\frac{1}{d_{\lambda_{0}}} \cdot \sum d_{\lambda} c_{\lambda}=1
$$

Therefore by (3.8)

$$
L\left(\frac{\chi_{0} \Re \lambda_{0}}{d_{\lambda_{0}}}\right)<1,
$$

which implies $\chi_{0}<\chi_{\lambda_{0}}$.

Corollary 3.10. There exists a simple root $\lambda_{0}$ such that $\chi_{\lambda_{0}}=\chi_{0}$.

We conclude that to compute $\chi_{0}$ it suffices to consider only simple roots:

$$
\chi_{0}=\min _{\lambda \in \Delta_{1}}\left\{\chi_{\lambda}\right\} .
$$

\subsection{Main theorems}

For any root $\lambda$ and $j \leqslant i_{\lambda}$, let $V_{\lambda, j}$ be the real subspace on $\mathcal{N}$ spanned by $Z_{\lambda, j}$ if $\lambda$ is real and by $\mathfrak{R} Z_{\lambda, j}$ and $\Im Z_{\lambda, j}$, otherwise. Then for $X \in \mathcal{N}$, by $\left.X\right|_{V_{\lambda, j}}$ we shall denote the projection of $X$ on $V_{\lambda, j}$.

Now we can state the main results of the paper

\section{Main Theorem A. Assume}

(A1) $\mathbb{E} \log M \in \mathcal{A}^{--}$;

(A2) for any root $\lambda$ there exists a positive number $\chi_{\lambda}$ such that $\mathbb{E}\left[\mathrm{e}^{\frac{\chi_{\lambda} \Re \lambda(\log M)}{d_{\lambda}}}\right]=1$;

(A3) the Laplace transform of the measure $\mu_{A}$ is finite in some neighborhood $U$ of 0 in $\mathcal{A}^{*}$ i.e. if $\alpha \in U$, then $L(\alpha)<\infty$

(A4) $\mathbb{E}|Q|^{\chi_{0}}<\infty$, for $\chi_{0}$ defined in (3.11).

Assume moreover that there exists a simple root $\lambda_{0}$ such that $\chi_{\lambda_{0}}=\chi_{0}$ satisfying

(A5) the law of $\Re \lambda_{0}(\log M)$ is non-arithmetic;

(A6) $\mathbb{E}\left[\mathrm{e}^{\frac{\chi_{\lambda_{0}} \mathfrak{R} \lambda_{0}(\log M)}{d_{\lambda_{0}}}}\left|\mathfrak{R} \lambda_{0}(\log M)\right|\right]<\infty$;

(A7) for any $X \in V_{\lambda_{0}, i_{\lambda_{0}}}$

$$
\mathbb{P}\left[\left.\log ((Q, M) \circ \exp X)\right|_{V_{\lambda_{0}, i_{\lambda_{0}}}}=X\right]<1 .
$$

Then there exists a positive constant $C_{1}$ and for any $\varepsilon>0$ there exists $C_{\varepsilon}$ such that

$$
C_{1} t^{-\chi_{0}} \leqslant \mathbb{P}[|R|>t] \leqslant C_{\varepsilon} t^{-\left(\chi_{0}-\varepsilon\right)} .
$$

A simple root $\lambda_{0}$ is called dominant if

$$
\chi_{\lambda_{0}}=\chi_{0}
$$


and if $\chi_{\lambda}=\chi_{0}$ for some other root $\lambda$, then there exists a constant $c_{\lambda}$ larger than 1 such that $\lambda=c_{\lambda} \lambda_{0}$.

Of course it may happen that dominant root does not exists, i.e. for two different simple roots $\lambda_{1}, \lambda_{2}$, such that $\lambda_{1} \neq c \lambda_{2}$ for any constant $c$, we have $\chi_{0}=\chi_{\lambda_{1}}=\chi_{\lambda_{2}}$.

Main Theorem B. Assume that the action of $A$ on $N$ is diagonalizable and

(B1) $\mathbb{E} \log M \in \mathcal{A}^{--}$;

(B2) for any root $\lambda$ there exists a positive number $\chi_{\lambda}$ such that $\mathbb{E}\left[\mathrm{e}^{\frac{\chi_{\lambda} \lambda(\log M)}{d_{\lambda}}}\right]=1$;

(B3) for any root $\lambda, \mathbb{E}\left[\mathrm{e}^{\frac{\chi_{\lambda} \lambda(\log M)}{d_{\lambda}}}|\lambda(\log M)|\right]<\infty$;

(B4) $\mathbb{E}|Q|^{\chi_{0}}<\infty$;

where $\chi_{0}$ was defined in (3.11). Assume moreover that there exists a simple root $\lambda_{0}$ such that $\chi_{\lambda_{0}}=\chi_{0}$ satisfying

(B5) the law of $\lambda_{0}(\log M)$ is non-arithmetic;

(B6) there exists $i \leqslant i_{\lambda_{0}}$ such that for every $X \in V_{\lambda_{0}, i}, \mathbb{P}\left[\left.\log ((Q, M) \circ \exp X)\right|_{V_{\lambda_{0}, i}}=X\right]<1$.

Then there exists a positive number $C_{1}$ such that

$$
\frac{1}{C_{1}} t^{-\chi_{0}} \leqslant \mathbb{P}[|R|>t] \leqslant C_{1} t^{-\chi_{0}} .
$$

Moreover if there exists in $\Delta_{1}$ a dominant root $\lambda_{0}$ satisfying both (B5) and (B6), then

$$
\lim _{t \rightarrow \infty} t^{\chi} \mathbb{P}[|R|>t]=C_{2},
$$

for some positive number $C_{2}$.

\section{Proof of Main Theorem A}

\subsection{Upper estimates}

In order to prove the upper bound of the tail of $R$, we shall use Lemma 2.19 and prove existence of $\chi$ th moment of $R$ for any $\chi$ satisfying $0<\chi<\chi_{0}$ and then the estimates follows immediately (Corollary 4.9).

Lemma 4.1. Under the hypothesis (A1)-(A4) the stationary measure of $R$ has all moments smaller than $\chi_{0}$, i.e.

$$
\mathbb{E}|R|^{\chi}<\infty
$$

for all $\chi$ satisfying $0<\chi<\chi_{0}$.

Proof. Fix $\chi^{\prime}$ such that $\chi<\chi^{\prime}<\chi_{0}$, then by definition of $\chi_{0}$

$$
L\left(\frac{\chi^{\prime} \mathfrak{R} \lambda}{d_{\lambda}}\right)<1, \quad \lambda \in \Delta
$$

For any root $\lambda$ let us define a positive number

$$
a_{\lambda}= \begin{cases}\chi, & \text { if } L\left(\frac{\chi \Re \lambda}{d_{\lambda}}\right)>L\left(\frac{\chi^{\prime} \Re \lambda}{d_{\lambda}}\right), \\ \chi^{\prime}, & \text { otherwise. }\end{cases}
$$

Then, since the Laplace transform is convex

$$
L\left(\frac{\beta \Re \lambda}{d_{\lambda}}\right)<L\left(\frac{a_{\lambda} \mathfrak{R} \lambda}{d_{\lambda}}\right)
$$

for any root $\lambda$ and $\beta \in\left(\chi, \chi^{\prime}\right)$. We may choose positive $\delta$ satisfying

$$
0<\delta<\frac{1}{L\left(a_{\lambda} \Re \lambda / d_{\lambda}\right)}-1, \quad \text { for any } \lambda \in \Delta .
$$


Consider the function

$$
f(s)=\mathbb{E}\left[\mathrm{e}^{s \sum_{i}\left|t_{i}(\log M)\right|}\right] .
$$

For any sequence $\sigma$ of 0 and 1's having the length $d$ define the element of $\mathcal{A}^{*}$ by the formula

$$
\alpha_{\sigma}(H)=\sum_{i=1}^{d}(-1)^{\sigma(i)} t_{i}(H), \quad H \in \mathcal{A},
$$

and notice that $f$ can be dominated by the sum

$$
f(s) \leqslant \sum_{\sigma \in\{0,1\}^{d}} L\left(s \alpha_{\sigma}\right) .
$$

By (A3) for small values of $s$ the Laplace transform $L\left(s t_{i}\right)$ is well-defined, moreover it is continuous as a function of $s$ and tends to 1 as $t$ goes to 0 . Therefore also $f$ is continuous and tends to 1 . So, there exists $\theta$, such that

$$
f(s)<1+\delta, \quad \text { for } s \leqslant \theta .
$$

Next, choose a positive number $\varepsilon$ satisfying

$$
\varepsilon<\min \left\{\frac{\theta\left(\chi^{\prime}-\chi\right)}{\chi \chi^{\prime}}, \frac{\theta}{2 \chi}\right\}
$$

Finally, define

$$
q=\frac{\theta}{\varepsilon \chi}, \quad p=\frac{\theta}{\theta-\varepsilon \chi} .
$$

Then notice that $\frac{1}{p}+\frac{1}{q}=1$, by (4.5)

$$
q>2 \text { and } p<2
$$

and moreover

$$
\chi<p \chi<\chi^{\prime}
$$

Recall that $R$ was constructed as the limit in distribution of $R_{n}$. Therefore it is enough to estimate $\chi$ th moment of $R_{n}$ independently on $n$. We have

$$
\begin{aligned}
\left(\mathbb{E}\left|R_{n}\right|^{\chi}\right)^{\frac{1}{\chi}} & =\left[\mathbb{E}\left|Q_{n} \cdot \delta_{M_{n}}\left(Q_{n-1}\right) \cdots \delta_{M_{1} \ldots M_{n}}\left(Q_{0}\right)\right|^{\chi}\right]^{\frac{1}{\chi}} \\
& \leqslant\left(\mathbb{E}\left[\sum_{k=0}^{n-1}\left\|M_{k+1} \cdots M_{n}\right\|\left|Q_{k}\right|\right]^{\chi}\right)^{\frac{1}{\chi}}+\left(\mathbb{E}\left|Q_{n}\right|^{\chi}\right)^{\frac{1}{\chi}} \\
& \leqslant \sum_{k=0}^{n-1}\left(\mathbb{E}\left[\left\|M_{k+1} \cdots M_{n}\right\|\left|Q_{k}\right|\right]^{\chi}\right)^{\frac{1}{\chi}}+\left(\mathbb{E}\left|Q_{n}\right|^{\chi}\right)^{\frac{1}{\chi}} \\
& \leqslant\left(\mathbb{E}|Q|^{\chi}\right)^{\frac{1}{\chi}}\left(1+\sum_{k=1}^{\infty}\left(\mathbb{E}\left\|M_{1} \cdots M_{k}\right\|^{\chi}\right)^{\frac{1}{\chi}}\right) .
\end{aligned}
$$

Thus, we have to prove that the series

$$
\sum_{k=1}^{\infty}\left(\mathbb{E}\left\|M_{1} \cdots M_{k}\right\|^{\chi}\right)^{\frac{1}{\chi}}
$$

is convergent.

For this purpose, observe that by Lemma 2.19, the Hölder inequality and (4.6) 


$$
\begin{aligned}
\mathbb{E}\left\|M_{1} \cdots M_{k}\right\|^{\chi} & \leqslant C \mathbb{E}\left[\max _{\lambda}\left\{\mathrm{e}^{\frac{\chi \Re \lambda\left(\log \Pi_{k}\right)}{d_{\lambda}}}\right\} \cdot\left(1 \vee \max _{i}\left|t_{i}\left(\log \Pi_{k}\right)\right|^{\chi D}\right)\right] \\
& \leqslant C\left(\mathbb{E}\left[\max _{\lambda}\left\{\mathrm{e}^{\frac{p \chi \Re \lambda\left(\log \Pi_{k}\right)}{d_{\lambda}}}\right\}\right]\right)^{\frac{1}{p}} \cdot\left(\mathbb{E}\left[1 \vee \max _{i}\left|t_{i}\left(\log \Pi_{k}\right)\right|^{q \chi D}\right]\right)^{\frac{1}{q}} \\
& \leqslant C^{\prime}\left(\mathbb{E}\left[\sum_{\lambda} \mathrm{e}^{\frac{p \chi \Re \lambda\left(\log \Pi_{k}\right)}{d_{\lambda}}}\right]\right)^{\frac{1}{p}} \cdot\left(\mathbb{E}\left[\prod_{i} \mathrm{e}^{\varepsilon q \chi \sum_{j \leqslant k}\left|t_{i}\left(\log M_{j}\right)\right|}\right]\right)^{\frac{1}{q}} \\
& \leqslant C^{\prime \prime} \sum_{\lambda}\left(\mathbb{E}\left[\mathrm{e}^{\frac{p \chi \Re \lambda(\log M)}{d_{\lambda}}}\right]\right)^{\frac{k}{p}} \cdot\left(\mathbb{E}\left[\mathrm{e}^{\varepsilon q \chi \sum_{i}\left|t_{i}(\log M)\right|}\right]\right)^{\frac{k}{q}} .
\end{aligned}
$$

Therefore, applying (4.6), (4.8) and (4.2) we obtain

$$
\begin{aligned}
\sum_{k=1}^{\infty}\left(\mathbb{E}\left\|M_{1} \cdots M_{k}\right\|^{\chi}\right)^{\frac{1}{\chi}} & \leqslant C \sum_{k=1}^{\infty}\left(\sum_{\lambda} L\left(\frac{p \chi \Re \lambda}{d_{\lambda}}\right)^{\frac{k}{p}} \cdot f(\varepsilon q \chi)^{\frac{k}{q}}\right)^{\frac{1}{\chi}} \\
& \leqslant C^{\prime} \sum_{\lambda} \sum_{k=1}^{\infty}\left[L\left(\frac{a_{\lambda} \Re \lambda}{d_{\lambda}}\right)^{\frac{1}{p}} \cdot(1+\delta)^{\frac{1}{q}}\right]^{\frac{k}{\chi}} \\
& \leqslant C^{\prime} \sum_{\lambda} \sum_{k=1}^{\infty}\left[\left(L\left(\frac{a_{\lambda} \Re \lambda}{d_{\lambda}}\right) \cdot(1+\delta)\right)^{\frac{1}{2 \chi}}\right]^{k},
\end{aligned}
$$

where for the last inequality we used (4.7).

Finally by (4.3)

$$
\left(L\left(\frac{a_{\lambda} \Re \lambda}{d_{\lambda}}\right) \cdot(1+\delta)\right)^{\frac{1}{2 \chi}}<1,
$$

therefore the series above converges.

Corollary 4.9. For any $\varepsilon$ there exists $C_{\varepsilon}$ such that

$$
\mathbb{P}[|R|>t] \leqslant C_{\varepsilon} t^{-\left(\chi_{0}-\varepsilon\right)}
$$

Proof. We have

$$
t^{\chi_{0}-\varepsilon} \mathbb{P}[|R|>t] \leqslant \int_{\{x:|x|>t\}}|x|^{\chi_{0}-\varepsilon} \nu(\mathrm{d} x) \leqslant \mathbb{E}|R|^{\chi_{0}-\varepsilon}
$$

and by the lemma above the value is finite.

\subsection{Lower estimates}

To prove the lower estimate we choose a simple root $\lambda_{0}$ such that $\chi_{\lambda_{0}}=\chi_{0}$, satisfying (A5)-(A7), and then study projection of the random walk $R_{n}$ on a suitable one or two dimensional linear subspace of $\mathcal{N}_{\lambda_{0}}$ or $\mathcal{N}_{\lambda_{0}}^{\mathbb{C}} \oplus \mathcal{N} \mathcal{\lambda}_{\bar{\lambda}_{0}}^{\mathbb{C}}$, respectively, depending whether $\lambda_{0}$ is real or complex. In both cases the projected random walk can be explicitly computed. If $\lambda_{0}$ is real we obtain just a random walk on $\mathbb{R}$ generated by the action of "ax $+b$ " group on $\mathbb{R}$, described in Section 3.2, and we conclude the result from Theorem 3.4. The case when $\lambda_{0}$ is complex is more complicated. Then we obtain a random walk on $\mathbb{R}^{2}$ generated by the action of $\mathbb{R}^{+} \times O(2)$ on $\mathbb{R}^{2}$, as studied in [2]. But our assumptions are different and we cannot apply the results proved there, so we shall give here a complete proof based on some ideas of A.K. Grincevičius [13] and Ch. Goldie [11].

Fix a simple root $\lambda_{0}$ satisfying all the assumptions (A1)-(A7). We shall consider two cases.

Case I. $\lambda_{0}$ is real.

Then we have the following lemma. 
Lemma 4.10. If $\lambda_{0}$ is real then there exists a positive constant $C$ such that

$$
\mathbb{P}[|R|>t] \geqslant C t^{-\chi_{0}} \text {. }
$$

Proof. Notice that by (2.8)

$$
\left(\delta_{a}(x)\right)_{\lambda_{0}, i_{\lambda_{0}}}=\mathrm{e}^{\lambda_{0}(\log a)} x_{\lambda_{0}, i_{\lambda_{0}}},
$$

for $x=\exp \left(\sum x_{\lambda, i} Z_{\lambda, i}\right)$. We shall prove that

$$
|x| \geqslant\left(\frac{\left|x_{\lambda_{0}, i_{\lambda_{0}}}\right|}{c_{\lambda_{0}, i_{\lambda_{0}}}}\right)^{\frac{1}{d_{\lambda_{0}}}} \quad \text { for } x \in N .
$$

Note that if for some $x \in N$

$$
\left|x_{\lambda_{0}, i_{\lambda_{0}}}\right| \geqslant c_{\lambda_{0}, 1} t^{d_{\lambda_{0}}}
$$

then

$$
\left|\left(\sigma_{t^{-1}}(x)\right)_{\lambda_{0}, i_{\lambda_{0}}}\right|=\left|\left(\delta_{\left(\exp (-\log t) H_{0}\right)}(x)\right)_{\lambda_{0}, i_{\lambda_{0}}}\right|=t^{-d_{\lambda_{0}}}\left|x_{\lambda_{0}, i_{\lambda_{0}}}\right| \geqslant c_{\lambda_{0}, i_{\lambda_{0}}}
$$

hence $|x| \geqslant t$, which gives (4.11).

Thus, we have

$$
\mathbb{P}[|R|>t] \geqslant \mathbb{P}\left[\left|R_{\lambda_{0}, i_{\lambda_{0}}}\right|>c_{\lambda_{0}, i_{\lambda_{0}}} t^{d_{\lambda_{0}}}\right] .
$$

But notice that because $\lambda_{0}$ is simple, in view of (2.13), we have

$$
\left(R_{n}\right)_{\lambda_{0}, i_{\lambda_{0}}}=\bar{M}_{n}\left(R_{n-1}\right)_{\lambda_{0}, i_{\lambda_{0}}}+\bar{Q}_{n},
$$

where

$$
\begin{aligned}
& \bar{M}_{n}=\mathrm{e}^{\lambda_{0}\left(\log M_{n}\right)}, \\
& \bar{Q}_{n}=\left(Q_{n}\right)_{\lambda_{0}, i_{\lambda_{0}}} .
\end{aligned}
$$

The foregoing formula defines a random walk on $\mathbb{R}$, generated by the action of the "ax $+b$ " group on $\mathbb{R}$, which is a special case of the situation described in Section 3.2. Moreover all the assumptions of Theorem (3.4) are satisfied for $\bar{\chi}=\chi_{0} / d_{\lambda_{0}}$ :

$$
\mathbb{E} \bar{M}^{\bar{\chi}}=\mathbb{E}\left[\mathrm{e}^{\frac{\chi_{0} \lambda_{0}(\log M)}{d_{\lambda_{0}}}}\right]=1
$$

and by (4.11)

$$
\mathbb{E}|\bar{Q}|^{\bar{\chi}} \leqslant C \mathbb{E}|Q|^{\chi_{0}}<\infty .
$$

$R_{\lambda_{0}, i_{\lambda_{0}}}$ is the limit in law of $\left(R_{n}\right)_{\lambda_{0}, i_{\lambda_{0}}}$, therefore there exists a positive constant $C$ such that

$$
\mathbb{P}\left[\left|R_{\lambda_{0}, i_{\lambda_{0}}}\right|>c_{\lambda_{0}, i_{\lambda_{0}}} t^{d_{\lambda_{0}}}\right] \geqslant C t^{-\bar{\chi} \cdot d_{\lambda_{0}}}=C t^{-\chi_{0}} .
$$

Combining the inequality above with (4.12) we obtain the lemma.

Case II. $\lambda_{0}$ is complex.

To simplify our notation we shall write $Z$ instead of $Z_{\lambda_{0}, i_{\lambda_{0}}}$, then $\bar{Z}=Z_{\bar{\lambda}_{0}, i_{\lambda_{0}}}$. Define

$$
\begin{aligned}
& X=\frac{1}{2}(Z+\bar{Z}), \\
& Y=-\frac{\mathrm{i}}{2}(Z-\bar{Z}) .
\end{aligned}
$$

For any $x \in N$ let $\left.x\right|_{V}$ denotes the projection of $\log x$ onto the real space $V$, spanned by $X$ and $Y$. Let $|\cdot|_{0}$ be the usual Euclidean norm on $V$, i.e. $|v|_{0}=\sqrt{\alpha^{2}+\beta^{2}}$ for $v=\alpha X+\beta Y \in V$. 
Lemma 4.13. We have

$$
\mathbb{P}[|R|>t] \geqslant \mathbb{P}\left[\left.|R|_{V}\right|_{0} \geqslant 2 c_{\lambda_{0}, i_{\lambda_{0}}} t^{d_{\lambda_{0}}}\right] .
$$

Proof. It is enough to prove that for any $x \in N$

$$
|x| \geqslant\left(\frac{\left.|x|_{V}\right|_{0}}{2 c_{\lambda_{0}, i_{\lambda_{0}}}}\right)^{\frac{1}{d_{\lambda_{0}}}} .
$$

Assume

$$
\left.|x|_{V}\right|_{0} \geqslant 2 c_{\lambda_{0}, i_{\lambda_{0}}} \cdot t^{d_{\lambda_{0}}} \text { and }\left.\quad x\right|_{V}=\alpha X+\beta Y .
$$

By (2.8) we have

$$
\begin{aligned}
& \delta_{a}(\exp Z)=\exp \left(\mathrm{e}^{\lambda_{0}(\log a)} Z+W\right), \\
& \delta_{a}(\exp \bar{Z})=\exp \left(\mathrm{e}^{\bar{\lambda}_{0}(\log a)} \bar{Z}+\bar{W}\right),
\end{aligned}
$$

where $W \in \operatorname{span}\left\{Z_{\lambda_{0}, 1}, \ldots, Z_{\lambda_{0}, i_{\lambda_{0}}-1}\right\}$.

Then

$$
\begin{aligned}
\sigma_{t^{-1}}(x) & =\sigma_{t^{-1}}\left(\exp \left(\frac{\alpha-\mathrm{i} \beta}{2} \cdot Z+\frac{\alpha+\mathrm{i} \beta}{2} \cdot \bar{Z}+W^{\prime}\right)\right) \\
& =\exp \left(\frac{\alpha-\mathrm{i} \beta}{2} \cdot t^{-\lambda_{0}\left(H_{0}\right)} Z+\frac{\alpha+\mathrm{i} \beta}{2} \cdot t^{-\lambda_{0}\left(H_{0}\right)} \bar{Z}+W^{\prime \prime}\right) .
\end{aligned}
$$

Notice that

$$
\left|\frac{\alpha-\mathrm{i} \beta}{2} \cdot t^{-\lambda_{0}\left(H_{0}\right)}\right|=\frac{\left.|x|_{V}\right|_{0}}{2} \cdot t^{-d_{\lambda_{0}}} \geqslant c_{\lambda_{0}, i_{\lambda_{0}}},
$$

therefore $\log \sigma_{t^{-1}}(x) \notin \Omega$, which implies $|x| \geqslant t$ and proves (4.14).

The lemma reduces the problem to prove existence of a positive constant $C$ such that

$$
\mathbb{P}\left[\left.|R|_{V}\right|_{0} \geqslant t\right] \geqslant C t^{-\frac{\chi_{0}}{d_{\lambda_{0}}}} .
$$

Let us restrict the random walk $R_{n}$ to the linear space $V$, defining $\bar{R}_{n}=\left.R_{n}\right|_{V}$. Then the following holds

Lemma 4.17. The random variables $\bar{R}_{n}$ satisfy the following stochastic recursion

$$
\bar{R}_{n}=\bar{M}_{n} \bar{R}_{n-1}+\bar{Q}_{n},
$$

where $\bar{Q}_{n}=\left.Q_{n}\right|_{V}$ and $\bar{M}_{n}=r\left(\bar{M}_{n}\right) O\left(\bar{M}_{n}\right)$, where

$$
r\left(\bar{M}_{n}\right)=\mathrm{e}^{\Re \lambda_{0}\left(\log M_{n}\right)}
$$

is an element of one parameter group of dilations of $\mathbb{R}^{2}$, and

$$
O\left(\bar{M}_{n}\right)=\left(\begin{array}{cc}
\cos \left(\Im \lambda_{0}\left(\log M_{n}\right)\right) & \sin \left(\Im \lambda_{0}\left(\log M_{n}\right)\right) \\
-\sin \left(\Im \lambda_{0}\left(\log M_{n}\right)\right) & \cos \left(\Im \lambda_{0}\left(\log M_{n}\right)\right)
\end{array}\right)
$$

belongs to the orthogonal group $O(2)$.

Proof. By (4.15)

$$
\begin{aligned}
\delta_{a}(\exp X)+\mathrm{i} \delta_{a}(\exp Y)= & \delta_{a}(\exp Z)=\exp \left(\mathrm{e}^{\lambda_{0}(\log a)} Z+W\right) \\
= & \exp \left(\mathrm{e}^{\Re \lambda_{0}(\log a)}\left(\cos \left(\Im \lambda_{0}(\log a)\right) X-\sin \left(\Im \lambda_{0}(\log a)\right) Y\right)\right. \\
& \left.+\mathrm{ie}^{\Re \lambda_{0}(\log a)}\left(\cos \left(\Im \lambda_{0}(\log a)\right) Y+\sin \left(\Im \lambda_{0}(\log a)\right) X\right)+W\right) .
\end{aligned}
$$


Since the action of $A$ is real we have

$$
\begin{aligned}
& \left.\delta_{a}(\exp X)\right|_{V}=\mathrm{e}^{\Re \lambda_{0}(\log a)}\left(\cos \left(\Im \lambda_{0}(\log a)\right) X-\sin \left(\Im \lambda_{0}(\log a)\right) Y\right), \\
& \left.\delta_{a}(\exp Y)\right|_{V}=\mathrm{e}^{\Re \lambda_{0}(\log a)}\left(\cos \left(\Im \lambda_{0}(\log a)\right) Y+\sin \left(\Im \lambda_{0}(\log a)\right) X\right) .
\end{aligned}
$$

which, in view of (2.13), implies the lemma.

Denote $\bar{S}=V \rtimes\left(\mathbb{R}^{+} \times O(2)\right)$ to be the semi-direct product of $V$ and $\mathbb{R}^{+} \times O(2)$, then $\bar{R}_{n}$ define a random walk on $V$ analogous to (3.1) i.e. $\bar{R}_{n}$ is a projection onto $V$ of $\left(\bar{Q}_{n}, \bar{M}_{n}\right) \cdots\left(\bar{Q}_{1}, \bar{M}_{1}\right)$ where $\left(\bar{Q}_{n}, \bar{M}_{n}\right)$ are i.i.d. $\bar{S}$-valued random variables.

Projecting our assumptions (A1)-(A7) onto $V$ we obtain

- $\mathbb{E} \log r(\bar{M})<0$,

- $\mathbb{E} r(\bar{M})^{\bar{\chi}}=1$, for $\bar{\chi}=\frac{\chi_{0}}{d_{\lambda_{0}}}$,

- $\mathbb{E}\left[r(\bar{M})^{\bar{\chi}}|\log r(\bar{M})|\right]<\infty$,

- $\mathbb{E}|\bar{Q}|_{0}^{\bar{\chi}}<\infty$.

Moreover $\bar{R}=\left.R\right|_{V}$ is the limit in distribution of $\bar{R}_{n}$. Random walks of this type were studied in [2], where asymptotic of their tails has been described:

$$
\lim _{t \rightarrow \infty} t^{\bar{x}} \mathbb{P}\left[|\bar{R}|_{0}>t\right]=C .
$$

In order to prove positivity of the constant $C$ the authors needed some additional hypothesis: boundedness of $|\bar{Q}|_{0}$ and larger moments of $r(\bar{M})$. The argument used there, based on a theorem of Landau, cannot be applied here. To prove positivity of $C$ we shall apply to our settings an approach of Grincevičius [13] and Goldie [11], who considered the problem on the "ax $+b$ " group.

Define the "backward" process $\bar{R}_{n}^{*}$ :

$$
\begin{aligned}
& \bar{R}_{0}^{*}=0, \\
& \bar{R}_{n}^{*}=\pi_{V}\left(\left(\bar{Q}_{1}, \bar{M}_{1}\right) \cdots\left(\bar{Q}_{n}, \bar{M}_{n}\right)\right)=\bar{Q}_{1}+\bar{\Pi}_{1} Q_{2}+\cdots+\bar{\Pi}_{n-1} \bar{Q}_{n},
\end{aligned}
$$

where

$$
\bar{\Pi}_{k}=\bar{M}_{1} \cdots \bar{M}_{k} .
$$

Recall that $\bar{R}_{n}^{*}$ converges pointwise to $\bar{R}$, and

$$
\bar{R}=\bar{R}_{n}^{*}+\bar{\Pi}_{n} \bar{R}^{*, n}
$$

where

$$
\bar{R}^{*, n}=\sum_{k=n+1}^{\infty}\left(\bar{M}_{n+1} \cdots \bar{M}_{k-1}\right) \bar{Q}_{k},
$$

hence for any $n, \bar{R}^{*, n}$ and $\bar{R}$ have the same distribution.

Lemma 4.19. There exists a positive constant $C$ such that

$$
\mathbb{P}\left[|\bar{R}|_{0}>t\right] \geqslant C t^{-\bar{\chi}} .
$$

Proof. Fix two positive numbers $\eta$ and $\delta$. There exists a ball $U$ in $V$ centered at some point $u$ of radius $\delta$ such that $\varepsilon=\mathbb{P}[\bar{R} \in U]$ is positive. Then by (4.18) we have

$$
\mathbb{P}\left[\inf _{x \in U}\left|\bar{R}_{n}^{*}+\bar{\Pi}_{n} x\right|_{0}>t \text { for some } n\right]=\sum_{n} \mathbb{P}\left[\max _{i<n} \inf _{x \in U}\left|\bar{R}_{i}^{*}+\bar{\Pi}_{i} x\right|_{0} \leqslant t \text { and } \inf _{x \in U}\left|\bar{R}_{n}^{*}+\bar{\Pi}_{n} x\right|_{0}>t\right]
$$




$$
\begin{aligned}
& =\frac{1}{\varepsilon} \sum_{n} \mathbb{P}\left[\max _{i<n} \inf _{x \in U}\left|\bar{R}_{i}^{*}+\bar{\Pi}_{i} x\right|_{0} \leqslant t \text { and } \inf _{x \in U}\left|\bar{R}_{n}^{*}+\bar{\Pi}_{n} x\right|_{0}>t\right] \mathbb{P}\left[\bar{R}^{*, n} \in U\right] \\
& =\frac{1}{\varepsilon} \sum_{n} \mathbb{P}\left[\max _{i<n} \inf _{x \in U}\left|\bar{R}_{i}^{*}+\bar{\Pi}_{i} x\right|_{0} \leqslant t \text { and } \inf _{x \in U}\left|\bar{R}_{n}^{*}+\bar{\Pi}_{n} x\right|_{0}>t \text { and }|\bar{R}|_{0}>t\right] \\
& \leqslant \frac{1}{\varepsilon} \mathbb{P}\left[|\bar{R}|_{0}>t\right] .
\end{aligned}
$$

Define

$$
U_{n}=\bar{R}_{n}^{*}+\bar{\Pi}_{n} u-\left(\bar{R}_{n-1}^{*}+\bar{\Pi}_{n-1} u\right)=\bar{\Pi}_{n-1}\left(\bar{Q}_{n}+\left(\bar{M}_{n}-I\right) u\right) .
$$

Then we have

$$
\begin{aligned}
\mathbb{P}\left[|\bar{R}|_{0}>t\right] & \geqslant \varepsilon \mathbb{P}\left[\inf _{x \in U}\left|\bar{R}_{n}^{*}+\bar{\Pi}_{n} x\right|_{0}>t \text { for some } n\right] \\
& \geqslant \varepsilon \mathbb{P}\left[\left|\bar{R}_{n}^{*}+\bar{\Pi}_{n} u\right|_{0}-r\left(\bar{\Pi}_{n}\right) \delta>t \text { for some } n\right] \\
& \geqslant \varepsilon \mathbb{P}\left[\left|U_{n}\right|_{0}-\left(r\left(\bar{\Pi}_{n}\right)+r\left(\bar{\Pi}_{n-1}\right)\right) \delta>2 t \text { for some } n\right] \\
& =\varepsilon \mathbb{P}\left[r\left(\bar{\Pi}_{n-1}\right)\left(\left|\bar{Q}_{n}+\left(\bar{M}_{n}-I\right) u\right|_{0}-\left(r\left(\bar{M}_{n}\right)+1\right) \delta\right)>2 t \text { for some } n\right] \\
& \geqslant \varepsilon \mathbb{P}\left[|\bar{Q}+(\bar{M}-I) u|_{0}-(r(\bar{M})+1) \delta>\eta\right] \mathbb{P}\left[\max _{n} r\left(\bar{\Pi}_{n}\right)>2 t / \eta\right] \\
& \geqslant C \mathbb{P}\left[|\bar{Q}+(\bar{M}-I) u|_{0}-(r(\bar{M})+1) \delta>\eta\right] t^{-\bar{\chi}},
\end{aligned}
$$

where the last inequality follows from (3.6). Finally we have to justify that for sufficiently small $\eta$ and $\delta$ the constant above is positive.

By (A7) there exist positive numbers $\eta, \theta$ such that

$$
\mathbb{P}\left[|\bar{Q}+(\bar{M}-I) u|_{0}>2 \eta\right]=\theta .
$$

Moreover by (A2) there is a large number $N$ such that

$$
\mathbb{P}[r(\bar{M}) \geqslant N] \leqslant \frac{\theta}{2},
$$

hence taking $\delta=\frac{\eta}{N+1}$ we obtain

$$
\begin{aligned}
\mathbb{P}\left[|\bar{Q}+(\bar{M}-I) u|_{0}-(r(\bar{M})+1) \delta>\eta\right] & \geqslant \mathbb{P}\left[|\bar{Q}+(\bar{M}-I) u|_{0}>2 \eta \text { and } r(\bar{M})<N\right] \\
& \geqslant \mathbb{P}\left[|\bar{Q}+(\bar{M}-I) u|_{0}>2 \eta\right]-\mathbb{P}[r(\bar{M}) \geqslant N] \\
& \geqslant \frac{\theta}{2},
\end{aligned}
$$

which finishes the proof.

Finally, in view of Lemma 4.13, the foregoing result implies the lower estimate of the tail of $R$ when $\lambda_{0}$ is complex.

\section{Proof of Main Theorem $B$}

\subsection{Diagonal action of $A$ on $N$}

In this section we shall change slightly our notation. From now we shall assume that the action of $A$ on $N$ is diagonalizable. Then all the roots are real and the real vectors $\left\{Z_{\lambda, j}\right\}_{\substack{\lambda \in i_{\lambda} \\ j \leqslant i_{\lambda}}}$ form a basis of $\mathcal{N}$. Let us denote these vectors by $X_{1}, \ldots, X_{n_{0}}\left(n_{0}=\operatorname{dim} N\right)$, then for any $H \in \mathcal{A}$

$$
\operatorname{ad}(H) X_{j}=\lambda_{j}(H) X_{j}, \quad j=1, \ldots, n_{0},
$$

for some root $\lambda_{j}$. In this notation it may of course happen that $\lambda_{i}=\lambda_{j}$ for $i \neq j$. 
Then the action of $A$ on $N$ is given by

$$
\delta_{a}(x)=\exp \left(\sum_{j} \mathrm{e}^{\lambda_{j}(H)} x_{j} X_{j}\right),
$$

for $x=\exp \left(\sum x_{j} X_{j}\right)$.

We change also numeration of constants defined in previous chapters. If $X_{j}=X_{\lambda, i}$ then we define

$$
\begin{aligned}
\chi_{j} & =\chi_{\lambda}, \\
c_{j} & =c_{\lambda, i}, \\
d_{j} & =d_{\lambda} .
\end{aligned}
$$

Notice that in this case both norms, on $N$ and on $A$, can be explicitly computed:

$$
\begin{aligned}
|x| & =\inf \left\{b: \log \left(\sigma_{b^{-1}}(x)\right) \in \Omega \cap \mathcal{N}\right\}=\inf \left\{b: \sum_{j} b^{-\lambda_{j}\left(H_{0}\right)} x_{j} X_{j} \in \Omega \cap \mathcal{N}\right\} \\
& =\inf \left\{b:\left|x_{j}\right|<c_{j} b^{d_{j}} \text { for } j=1, \ldots, n_{0}\right\}=\inf \left\{b: \frac{\left|x_{j}\right|^{\frac{1}{d_{j}}}}{c_{j}^{\frac{1}{d_{j}}}}<b\right\}=\max _{j}\left\{\bar{c}_{j}\left|x_{j}\right|^{\frac{1}{d_{j}}}\right\}
\end{aligned}
$$

for $\bar{c}_{j}=c_{j}^{-\frac{1}{d_{j}}}$, and next

$$
\|a\|=\max _{|x|=1}\left|\delta_{a}(x)\right|=\max _{j}\left\{\mathrm{e}^{\frac{\lambda_{j}(\log a)}{d_{j}}}\right\} .
$$

Let us define the lower central sequence in $\mathcal{N}$

$$
\begin{aligned}
& \mathcal{N}_{0}=\mathcal{N}, \\
& \mathcal{N}_{i+1}=\left[\mathcal{N}_{i}, \mathcal{N}\right],
\end{aligned}
$$

for $i=1, \ldots, m_{0}$ and $\mathcal{N}_{m_{0}+1}=\{0\}$. Then we may assume that there is a sequence $0=i_{0} \leqslant i_{1} \leqslant \cdots \leqslant i_{m_{0}}=n_{0}$ such that $X_{i_{j}+1}, \ldots, X_{i_{j+1}}$ are a basis of $\mathcal{N}_{j} / \mathcal{N}_{j+1}$.

Define

$$
I_{1}=\left\{1, \ldots, i_{1}\right\}
$$

and notice the set of indices of simple roots is a subset of $I_{1}$.

We shall use the lower central sequence to obtain a better description of the Campbell-Hausdorf formula [10]. If $(x \cdot y)_{i}$ denotes $i$ th coordinate of $x \cdot y$, for $x=\exp \left(\sum x_{i} X_{i}\right), y=\exp \left(\sum y_{i} X_{i}\right)$ elements of $N$, then

$$
\begin{aligned}
& (x \cdot y)_{i}=x_{i}+y_{i} \quad \text { for } i \in I_{1}, \\
& (x \cdot y)_{i}=x_{i}+y_{i}+P_{i}(x, y) \quad \text { for } i \in I_{p}, \text { for } p>1,
\end{aligned}
$$

where $P_{i}$ are polynomials depending on $x_{1}, \ldots x_{i_{p-1}}, y_{1}, \ldots y_{i_{p-1}}$ and they can be written as

$$
P_{i}(x, y)=\sum_{\underline{\mathbf{a}}, \underline{\mathbf{b}}} c_{\underline{\mathbf{a}}, \underline{\mathbf{b}}} P_{i}^{\mathbf{a}, \underline{\mathbf{b}}}(x, y)=\sum_{\underline{\mathbf{a}}, \underline{\mathbf{b}}} c_{\underline{\mathbf{a}}, \underline{\mathbf{b}}} x^{\underline{\mathbf{a}}} y^{\underline{\mathbf{b}}},
$$

where $c_{\underline{\mathbf{a}}, \underline{\mathbf{b}}}$ are some real constants (most of them are zero, then we assume $P_{j}^{\mathbf{a}, \underline{\mathbf{b}}}=0$, but for at least one pair $(\underline{\mathbf{a}}, \underline{\mathbf{b}}$ ) the constant $c_{\underline{\mathbf{a}}, \underline{\mathbf{b}}}$ is nonzero), $\underline{\mathbf{a}}$ and $\underline{\mathbf{b}}$ are multi-indexes of natural numbers of the length $i_{p-1}$, and we have used the notation (which will be used also in the rest of the paper without any saying):

- $0^{0}=1$;

- if $\underline{\mathbf{c}}$ is a multi-index of the length $i$ and $z$ is a vector of length at least $i$ (usually it will be longer than $i$ ) then

$$
z^{\underline{\mathbf{c}}}=\prod_{j \leqslant i} z_{j}^{\mathbf{c}_{j}}
$$


Moreover, we shall strongly rely on the following properties of the Campbell-Hausdorf formula: if $c_{\underline{\mathbf{a}}, \underline{\mathbf{b}}}$ is nonzero then:

both $\underline{\mathbf{a}}$ and $\underline{\mathbf{b}}$ are nonzero and $\sum_{j<i}\left(\underline{\mathbf{a}}_{j}+\underline{\mathbf{b}}_{j}\right) \lambda_{j}=\lambda_{i}$.

In order to prove the last equation we shall use (5.1). Fix $H \in \mathcal{A}$, then for any $x, y \in N$ we have

$$
\left(\delta_{\exp H}(x y)\right)_{i}=\mathrm{e}^{\lambda_{i}(H)}(x \cdot y)_{i},
$$

but on the other side, by (2.1) we write

$$
\begin{aligned}
\left(\delta_{\exp H}(x y)\right)_{i} & =\left(\delta_{\exp H}(x) \cdot \delta_{\exp H}(x)\right)_{i} \\
& =\sum_{\underline{\mathbf{a}}, \underline{\mathbf{b}}} c_{\underline{\mathbf{a}}, \underline{\mathbf{b}}}\left(\delta_{\exp H}(x)\right)^{\mathbf{a}}\left(\delta_{\exp H}(y)\right)^{\underline{\mathbf{b}}}=\sum_{\underline{\mathbf{a}}, \underline{\mathbf{b}}} c_{\underline{\mathbf{a}}, \underline{\mathbf{b}}} \mathrm{e}^{\left.\sum_{j<i} \underline{\mathbf{a}}_{j}+\underline{\mathbf{b}}_{j}\right) \lambda_{j}(H)} x \underline{\mathbf{a}} y \underline{\mathbf{b}} .
\end{aligned}
$$

Comparing last two equations we obtain (5.6).

\subsection{Proof of the first part of Main Theorem B}

To prove the theorem we shall compute explicitly $R$. Recall that $R$ is the pointwise limit of the "backward" process $R_{n}^{*}$, by definition (3.3) and the Campbell-Hausdorf formula (5.4) we have

$$
\begin{aligned}
\left(R_{n+1}^{*}\right)_{j} & =\left(R_{n}^{*} \cdot \delta_{\Pi_{n}}\left(Q_{n+1}\right)\right)_{j} \\
& =\left(R_{n}^{*}\right)_{j}+\mathrm{e}^{\lambda_{j}\left(\log \Pi_{n}\right)}\left(Q_{n+1}\right)_{j}+\sum_{\underline{\mathbf{a}, \underline{\mathbf{b}}}} c_{\underline{\mathbf{a}}, \underline{\mathbf{b}}}\left(R_{n}^{*}\right)^{\mathbf{a}}\left(\delta_{\Pi_{n}}\left(Q_{n+1}\right)\right)^{\underline{\mathbf{b}}} \\
& =\sum_{k=0}^{n} \mathrm{e}^{\lambda_{j}\left(\log \Pi_{k}\right)}\left(Q_{k+1}\right)_{j}+\sum_{\underline{\mathbf{a}}, \underline{\mathbf{b}}} c_{\underline{\mathbf{a}}, \underline{\mathbf{b}}} \sum_{k=0}^{n}\left(R_{k}^{*}\right)^{\mathbf{a}}\left(\delta_{\Pi_{k}}\left(Q_{k+1}\right)\right)^{\underline{\mathbf{b}}} .
\end{aligned}
$$

Hence

$$
\left(R_{n+1}^{*}\right)_{j}= \begin{cases}T_{n}^{j}, & \text { for } j \in I_{1}, \\ T_{n}^{j}+\sum_{\underline{\mathbf{a}}, \underline{\mathbf{b}}} c_{\mathbf{a}, \underline{\mathbf{b}}} \sum_{k=0}^{n}\left(R_{k}^{*}\right) \underline{\mathbf{a}}\left(\delta_{\Pi_{k}}\left(Q_{k+1}\right)\right) \underline{\mathbf{b}}, & \text { for } j \notin I_{1},\end{cases}
$$

where

$$
T_{n}^{j}=\sum_{k=0}^{n} \mathrm{e}^{\lambda_{j}\left(\log \Pi_{k}\right)}\left(Q_{k+1}\right)_{j} .
$$

Notice that $T_{n}^{j}$ is the "backward" process for a random walk generated by i.i.d. random variables $\left(\mathrm{e}^{\lambda_{j}\left(\log M_{k}\right)},\left(Q_{k}\right)_{j}\right)$, which converges pointwise to some random variable $T^{j}$. We shall later estimate $T_{n}^{j}$ by

$$
\bar{T}_{n}^{j}=\sum_{k=0}^{n} \mathrm{e}^{\lambda_{j}\left(\log \Pi_{k}\right)}\left|\left(Q_{k+1}\right)_{j}\right|,
$$

then $\bar{T}_{n}^{j}$ is also the "backward process" generated by $\left(\mathrm{e}^{\lambda_{j}\left(\log M_{k}\right)},\left|\left(Q_{k}\right)_{j}\right|\right) \cdot \bar{T}_{n}^{j}$ converges monotonously to a random variable $\bar{T}^{j}$, which by Theorem 3.4 satisfies

$$
\mathbb{P}\left[\bar{T}^{j}>t^{d_{j}}\right] \leqslant C t^{-\chi_{j}} .
$$

Lemma 5.9. For any $i$

$$
\mathbb{P}\left[\left|\left(R_{k}^{*}\right)_{i}\right|>t^{d_{i}} \text { for some } k\right] \leqslant C t^{-\chi_{0}} .
$$


Proof. For $j \in I_{1}$ we have

$$
\begin{aligned}
\mathbb{P}\left[\left|\left(R_{n}^{*}\right)_{j}\right|>t^{d_{j}} \text { for some } n\right] & =\mathbb{P}\left[\left|T_{n}^{j}\right|>t^{d_{j}} \text { for some } n\right] \leqslant \mathbb{P}\left[\bar{T}_{n}^{j}>t^{d_{j}} \text { for some } n\right] \\
& =\mathbb{P}\left[\bar{T}^{j}>t^{d_{j}}\right] \leqslant C t^{-\chi_{j}} \leqslant C t^{-\chi_{0}} .
\end{aligned}
$$

Fix $j \notin I_{1}$ and assume that the lemma holds for $i<j$. Then by (5.7), for $C=\sum\left|c_{\underline{\mathbf{a}}, \underline{\mathbf{b}}}\right|+1$, we have

$$
\begin{aligned}
& \mathbb{P}\left[\left|\left(R_{n+1}^{*}\right)_{j}\right|>t^{d_{j}} \text { for some } n\right]
\end{aligned}
$$

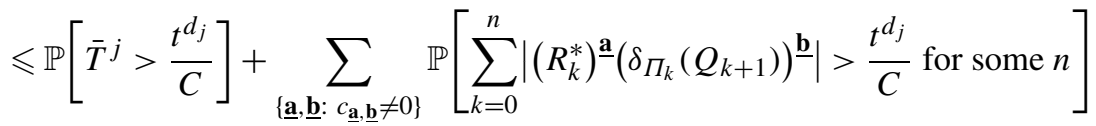

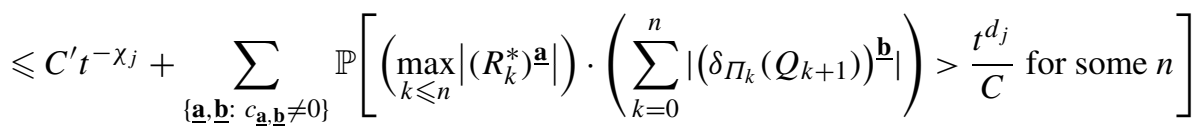

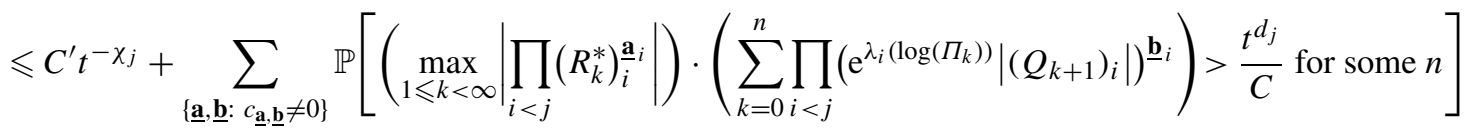

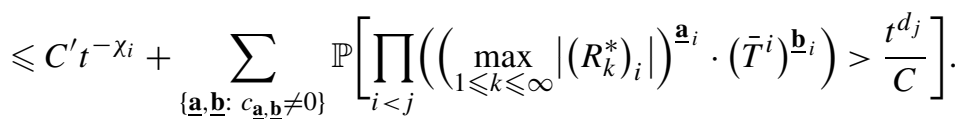

If $c_{\underline{\mathbf{a}}, \underline{\mathbf{b}}}$ is nonzero, then by (5.6) and (2.18)

$$
d_{j}=\sum_{i<j}\left(\underline{\mathbf{a}}_{i}+\underline{\mathbf{b}}_{i}\right) d_{i} .
$$

Therefore the above expression can be dominated by

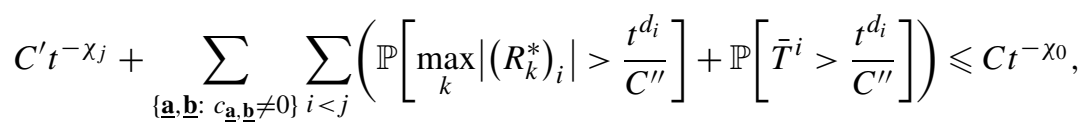

by the induction assumption and (5.8).

Corollary 5.13. There exists a positive constant $C$ such that

$$
\frac{1}{C} t^{-\chi_{0}} \leqslant \mathbb{P}[|R|>t] \leqslant C t^{-\chi_{0}} .
$$

Proof. By the lemma above we have

$$
\mathbb{P}[|R|>t] \leqslant \sum_{j} \mathbb{P}\left[\left|R_{j}\right|>c_{j} t^{d_{j}}\right] \leqslant \sum_{j} \mathbb{P}\left[\left|\left(R_{n}^{*}\right)_{j}\right| \geqslant c_{j} t^{d_{j}} \text { for some } n\right] \leqslant C t^{-\chi_{0}} .
$$

On the other hand, choose $j_{0}$ such that $\lambda_{j_{0}}$ is a simple root, $\chi_{j_{0}}=\chi_{0}$ and satisfies (A3) and (A4). Then by (5.2) and Theorem 3.4

$$
\mathbb{P}[|R|>t] \geqslant \mathbb{P}\left[\left|R_{j_{0}}\right| \geqslant c_{j_{0}} t^{d_{j_{0}}}\right] \geqslant \mathbb{P}\left[\left|T^{j_{0}}\right| \geqslant c_{j_{0}} t^{d_{j_{0}}}\right] \geqslant C t^{-\chi_{0}} .
$$

\subsection{Dominant root}

Now, we shall assume that there exists in $\Delta l_{1}$ a dominant root. Let us denote it by $\lambda_{0}$ and let $j_{0}$ be an index such that $\lambda_{j_{0}}=\lambda_{0}$. Define

$$
I_{0}=\left\{j: \lambda_{j} \text { is a multiple of } \lambda_{0}\right\} .
$$

Then by Lemma 3.9 


$$
\begin{aligned}
& \mathbb{E}\left[\mathrm{e}^{\frac{\chi_{0} \lambda_{j}(\log M)}{d_{j}}}\right]=1, \quad \text { for } j \in I_{0} \\
& \mathbb{E}\left[\mathrm{e}^{\frac{\chi_{0} \lambda_{j}(\log M)}{d_{j}}}\right]<1, \quad \text { for } j \notin I_{0} .
\end{aligned}
$$

Let $\mathcal{N}_{0}$ be the Lie algebra defined by

$$
\mathcal{N}_{0}=\text { Lie } \operatorname{span}\left\{X_{j}\right\}_{j \in I_{0}}=\bigoplus_{\left\{\lambda: \lambda \text { is a multiple of } \lambda_{0}\right\}} \mathcal{N}_{\lambda} .
$$

For any $j \in I_{0}$ let $s_{j}$ be the unique number such that $\lambda_{j}=s_{j} \lambda_{0}$. Put $N_{0}=\exp \mathcal{N}_{0}$. Notice that for $x \in N_{0}$ and any $a \in A$, by (5.2)

$$
\delta_{a}(x)=\exp \left(\sum_{j \in I_{0}} \mathrm{e}^{\lambda_{j}(\log a)} x_{j} X_{j}\right)=\exp \left(\sum_{j \in I_{0}} b^{s_{j} \lambda_{0}\left(H_{0}\right)} x_{j} X_{j}\right)=\sigma_{b}(x),
$$

where $b=\mathrm{e}^{\frac{\lambda_{0}(\log a)}{\lambda_{0}\left(H_{0}\right)}}$, hence the action of $A$ on $N_{0}$ depends only on the value of $\lambda_{0}(\log a)$.

Let $S_{0}=N_{0} \rtimes \mathbb{R}^{+}$be the semi-direct product of $N_{0}$ and $\mathbb{R}^{+}$with the group multiplication

$$
(x, b) \cdot\left(x^{\prime}, b^{\prime}\right)=\left(x \cdot \sigma_{b}\left(x^{\prime}\right), b b^{\prime}\right), \quad x, x^{\prime} \in N_{0}, b, b^{\prime} \in \mathbb{R}^{+} .
$$

Denote by $|\cdot|_{0}$ the restriction of $|\cdot|$ to $N_{0}$, i.e. $|x|_{0}=|x|$ for $x \in N_{0}$, by (5.2)

$$
|x|_{0}=\max _{j \in I_{0}}\left\{\bar{c}_{j}\left|x_{j}\right|^{\frac{1}{d_{j}}}\right\} \text {. }
$$

For any $x=\exp \left(\sum x_{\lambda, j} X_{\lambda, j}\right) \in N$ let $\left.x\right|_{N_{0}}$ denotes its restriction to $N_{0}$, i.e.

$$
\left.x\right|_{N_{0}}=\exp \left(\sum_{j \in I_{0}} x_{j} X_{j}\right) \text {. }
$$

We shall prove two lemmas

Lemma 5.16. We have

$$
\lim _{t \rightarrow \infty} t^{\chi_{0}} \mathbb{P}\left[|\bar{R}|_{0}>t^{d_{\lambda_{0}}}\right]=C_{+},
$$

for some positive constant $C_{+}$, where $\bar{R}=\left.R\right|_{N_{0}}$.

Lemma 5.17. For any $j$ not being an element of $I_{0}$

$$
\lim _{t \rightarrow \infty} t^{\chi_{0}} \mathbb{P}\left[\left|R_{j}\right|^{\frac{1}{d_{j}}}>t\right]=0 .
$$

Notice, that the lemmas imply the second part of Main Theorem B.

Proof of Main Theorem B. We have

$$
\begin{aligned}
\mathbb{P}[|R|>t] & \leqslant \mathbb{P}\left[\max _{j \in I_{0}}\left\{\bar{c}_{j}\left|R_{j}\right|^{\frac{1}{d_{j}}}\right\}>t\right]+\mathbb{P}\left[\max _{j \notin I_{0}}\left\{\bar{c}_{j}\left|R_{j}\right|^{\frac{1}{d_{j}}}\right\}>t\right] \\
& \leqslant \mathbb{P}\left[|\bar{R}|_{0}>t\right]+\sum_{j \notin I_{0}} \mathbb{P}\left[\bar{c}_{j}\left|R_{j}\right|^{\frac{1}{d_{j}}}>t\right]
\end{aligned}
$$

hence

$$
\lim _{t \rightarrow \infty} t^{\chi_{0}} \mathbb{P}[|R|>t] \leqslant C_{+} .
$$

On the other hand

$$
\mathbb{P}[|R|>t] \geqslant \mathbb{P}\left[\max _{j \in I_{0}}\left\{\bar{c}_{j}\left|R_{j}\right|^{\frac{1}{d_{j}}}\right\}>t\right]=\mathbb{P}\left[|\bar{R}|_{0}>t\right],
$$


which gives

$$
\lim _{t \rightarrow \infty} t^{\chi} \mathbb{P}[|R|>t] \geqslant C_{+}
$$

\subsection{Proofs of Lemmas 5.16 and 5.17}

Lemma 5.18. For any $x, y \in N$

$$
\left.\left.x\right|_{N_{0}} \cdot y\right|_{N_{0}}=\left.(x \cdot y)\right|_{N_{0}} .
$$

Proof. Suppose $j \in I_{0}$, then by the Campbell-Hausdorf formula (5.4)

$$
(x \cdot y)_{j}=x_{j}+y_{j}+\sum c_{\underline{\mathbf{a}}, \underline{\mathbf{b}}} x^{\underline{\mathbf{a}}} y^{\underline{\mathbf{b}}} .
$$

To prove the Lemma we have to justify that in the formula above do not appear coordinates $x_{i}$ and $y_{i}$ for $i \notin I_{0}$. In other word we should infer that if $c_{\underline{\mathbf{a}}, \underline{\mathbf{b}}}$ is not zero and $\underline{\mathbf{a}}_{i}+\underline{\mathbf{b}}_{i} \neq 0$ for some index $i$, then $i$ belongs to the set $I_{0}$.

Assume $c_{\underline{\mathbf{a}}, \underline{\mathbf{b}}} \neq 0$. Then by (5.6)

$$
\lambda_{j}=\sum_{i<j}\left(\underline{\mathbf{a}}_{i}+\underline{\mathbf{b}}_{i}\right) \lambda_{i}
$$

$\lambda_{j}$ is a multiple of $\lambda_{0}$, therefore $\chi_{j}=\chi_{0}$. By Lemma 3.9, this implies that if $\underline{\mathbf{a}}_{i}+\underline{\mathbf{b}}_{i} \neq 0$ then $\chi_{i}=\chi_{0}$, but (5.14) says that then $\chi_{i}$ is a multiple of $\lambda_{0}$ and we conclude the proof.

Proof of Lemma 5.16. For random variable $\left(Q_{j}, M_{j}\right)$ as in (3.1) define

$$
\begin{aligned}
& \bar{Q}_{j}=\left.Q_{j}\right|_{N_{0}}, \\
& \bar{M}_{j}=\mathrm{e}^{\frac{\lambda_{0}\left(\log M_{j}\right)}{\lambda_{0}\left(H_{0}\right)}} .
\end{aligned}
$$

Then $\left(\bar{Q}_{j}, \bar{M}_{j}\right)$ are i.i.d. $S_{0}$-valued random variables and satisfy assumptions of Theorem 3.4. Consider the random walk on $S_{0}$

$$
\bar{S}_{n}=\left(\bar{Q}_{n}, \bar{M}_{n}\right) \cdots\left(\bar{Q}_{1}, \bar{M}_{1}\right),
$$

by Lemma (5.18) and (5.15)

$$
\bar{R}_{j}=\pi_{N_{0}}\left(\bar{S}_{n}\right)=\left.R_{j}\right|_{N_{0}},
$$

therefore $\bar{R}_{j}$ converges in law to $\bar{R}$, and we may apply Theorem 3.4, which finishes the proof.

Lemma 5.19. If $j$ does not belong to $I_{0}$, then there exists $\varepsilon>0$ such that

$$
\mathbb{P}\left[\left|\left(R_{k}^{*}\right)_{j}\right|>t^{d_{j}} \text { for some } k\right] \leqslant C t^{-\left(\chi_{0}+\varepsilon\right)} .
$$

Proof. The idea of the proof is the same as of Lemma 5.9, but now we shall proceed more delicate.

If $j \in I_{1}$, then by (5.10)

$$
\mathbb{P}\left[\left|\left(R_{k}^{*}\right)_{j}\right|>t^{d_{j}} \text { for some } k\right] \leqslant C t^{-\chi_{j}} .
$$

Next assume that $j \notin I_{1} \cup I_{0}$ and the Lemma holds for $i<j$. Then arguing as in (5.11) we obtain

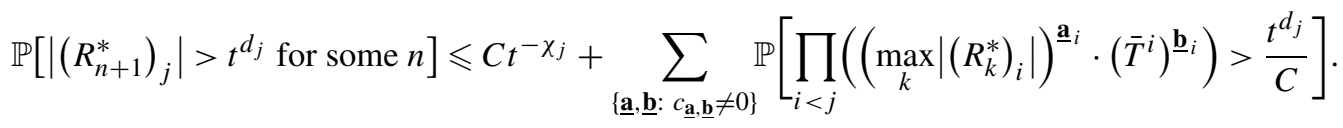

By (5.14) $\chi_{j}>\chi_{0}$, therefore it is enough to estimate, for any nonzero $c_{\mathbf{a}, \underline{\mathbf{b}}}$ the appropriate factor in the sum above.

The root $\lambda_{j}$ is not a multiple of $\lambda_{0}$, therefore, if $c_{\underline{\mathbf{a}}, \underline{\mathbf{b}}} \neq 0$, then by (5.6) there exists an index $i_{0}<j$ such that $\lambda_{i_{0}}$ is not a multiple of $\lambda_{0}$ and $\underline{\mathbf{a}}_{i_{0}}+\underline{\mathbf{b}}_{i_{0}}>0$. We shall consider two cases

Case 1. $\underline{\mathbf{a}}_{i_{0}}>0$. 
By the induction hypothesis there exists $\chi^{\prime}>\chi_{0}$ such that

$$
\mathbb{P}\left[\left|\left(R_{k}^{*}\right)_{i_{0}}\right|>t^{d_{i_{0}}} \text { for some } k\right] \leqslant C t^{-\chi^{\prime}} .
$$

Then take any positive number $\delta$ satisfying

$$
\delta<\frac{d_{i_{0}}\left(\chi^{\prime}-\chi_{0}\right)}{\chi^{\prime}}
$$

and define

$$
\delta^{\prime}=\frac{\delta \underline{\mathbf{a}}_{i_{0}}}{\sum_{i \neq i_{0}, i<j}\left(\underline{\mathbf{a}}_{i}+\underline{\mathbf{b}}_{i}\right)+\underline{\mathbf{b}}_{i_{0}}} .
$$

Then, in view of (5.12)

$$
d_{j}=\underline{\mathbf{a}}_{i_{0}}\left(d_{i_{0}}-\delta\right)+\sum_{i \neq i_{0}, i<j}\left(\underline{\mathbf{a}}_{i}+\underline{\mathbf{b}}_{i}\right)\left(d_{i}+\delta^{\prime}\right)+\underline{\mathbf{b}}_{i_{0}}\left(d_{i_{0}}+\delta^{\prime}\right)
$$

and by Lemma 5.9 and (5.20)

$$
\begin{aligned}
& \mathbb{P}\left[\prod_{i<j}\left(\left(\max _{k}\left|\left(R_{k}^{*}\right)_{i}\right|\right)^{\mathbf{a}_{i}} \cdot\left(\bar{T}^{i}\right)^{\underline{\mathbf{b}}_{i}}\right)>\frac{t^{d_{j}}}{C}\right] \\
& \leqslant \mathbb{P}\left[\max _{k}\left|\left(R_{k}^{*}\right)_{i_{0}}\right|>\frac{t^{d_{i_{0}}-\delta}}{C^{\prime}}\right]+\sum_{i \neq i_{0}, i<j} \mathbb{P}\left[\max _{k}\left|\left(R_{k}^{*}\right)_{i}\right|>\frac{t^{d_{i}+\delta^{\prime}}}{C^{\prime}}\right]+\sum_{i<j} \mathbb{P}\left[\bar{T}^{i}>\frac{t^{d_{i}+\delta^{\prime}}}{C^{\prime}}\right] \\
& \leqslant C\left(t^{-\chi^{\prime} \cdot \frac{d_{i_{0}}-\delta}{d_{i_{0}}}}+\sum_{i<j} t^{-\chi_{0} \cdot \frac{d_{i}+\delta^{\prime}}{d_{i}}}\right) \leqslant C t^{-\chi}
\end{aligned}
$$

for some $\chi>\chi_{0}$.

Case 2. $\underline{\mathbf{b}}_{i_{0}}>0$.

Then take $\delta$ as in (5.21) and define

$$
\delta^{\prime \prime}=\frac{\delta \underline{\mathbf{b}}_{i_{0}}}{\sum_{i \neq i_{0}, i<j}\left(\underline{\mathbf{a}}_{i}+\underline{\mathbf{b}}_{i}\right)+\underline{\mathbf{a}}_{i_{0}}} .
$$

Arguing as above we obtain

$$
\begin{aligned}
& \mathbb{P}\left[\prod_{i<j}\left(\left(\max _{k}\left|\left(R_{k}^{*}\right)_{i}\right|\right)^{\mathbf{a}_{i}} \cdot\left(\bar{T}^{i}\right)^{\underline{\mathbf{b}}_{i}}\right)>\frac{t^{d_{j}}}{C}\right] \\
& \leqslant \mathbb{P}\left[\bar{T}^{i_{0}}>\frac{t^{d_{i_{0}}-\delta}}{C^{\prime}}\right]+\sum_{i<j} \mathbb{P}\left[\max _{k}\left|\left(R_{k}^{*}\right)_{i}\right|>\frac{t^{d_{i}+\delta^{\prime \prime}}}{C^{\prime}}\right]+\sum_{i \neq i_{0}, i<j} \mathbb{P}\left[\bar{T}^{i}>\frac{t^{d_{i}+\delta^{\prime \prime}}}{C^{\prime}}\right] \\
& \leqslant C\left(t^{-\chi^{\prime} \cdot \frac{d_{i_{0}}-\delta}{d_{i_{0}}}}+\sum_{i<j} t^{-\chi_{0} \cdot \frac{d_{i}+\delta^{\prime \prime}}{d_{i}}}\right) \leqslant C t^{-\chi}
\end{aligned}
$$

for some $\chi>\chi_{0}$.

Proof of Lemma 5.17. The lemma is an immediate consequence of the above lemma.

\section{References}

[1] R. Azencott, E. Wilson, On homogeneous manifolds with negative curvature, part I, Trans. Amer. Math. Soc. 215 (1976) (1976) $323-362$.

[2] D. Buraczewski, E. Damek, Y. Guivarc'h, A. Hulanicki, R. Urban, On tail properties of stochastic recursions connected with generalized rigid motions, preprint. 
[3] D. Buraczewski, E. Damek, A. Hulanicki, Asymptotic behavior of Poisson kernels NA group, Comm. Partial Differential Equations 31 (2006) $1547-1589$.

[4] E. Damek, Left-invariant degenerate elliptic operators on semidirect extensions of homogeneous groups, Studia Math. 89 (1988) $169-196$.

[5] E. Damek, A. Hulanicki, Boundaries for leftinvariant subelliptic operators on semidirect products of nilpotent and Abelian groups, J. Reine Angew. Math. 411 (1990) 1-38.

[6] E. Damek, A. Hulanicki, Asymptotic behavior of the invariant measure for a diffusion related to a NA group, Colloq. Math. 104 (2006) 285-309.

[7] B. de Saporta, Y. Guivarc'h, E. Le Page, On the multidimensional stochastic equation $Y_{n+1}=A_{n} Y_{n}+B_{n}$, C. R. Math. Acad. Sci. Paris 339 (7) (2004) 499-502.

[8] J. Faraut, A. Korányi, Analysis on Symmetric Cones, Clarendon Press, Oxford, 1994.

[9] W. Feller, An Introduction to Probability Theory and its Application II, John Wiley and Sons, New York, 1966.

[10] G.B. Folland, E.M. Stein, Hardy Spaces on Homogeneous Groups, Princeton Univ. Press, Princeton, NJ, 1982.

[11] Ch.M. Goldie, Implicit renewal theory and tails of solutions of random equations, Ann. Appl. Probab. 1 (1) (1991) $126-166$.

[12] A.K. Grincevičius, On limit distribution for a random walk on the line, Lithuanian Math. J. 15 (1975) 580-589.

[13] A.K. Grincevičius, Products of random affine transformations, Lithuanian Math. J. 20 (1980) 279-282.

[14] Y. Guivarc'h, Heavy tail properties of multidimensional stochastic recursions, in: Dynamics \& Stochastic, in: IMS Lecture Notes Monograph Series, vol. 48, 2006, pp. 85-99.

[15] W. Hebisch, A. Sikora, A smooth subadditive homogeneous norm on a homogeneous group, Studia Math. 96 (3) (1990) $231-236$.

[16] E. Heintze, On homogeneous manifolds of negative curvature, Math. Ann. 211 (1974) 23-34.

[17] H. Kesten, Random difference equations and renewal theory for products of random matrices, Acta Math. 131 (1973) $207-248$.

[18] C. Klüppelberg, S. Pergamenchtchikov, The tail of the stationary distribution of a random coefficient AR(q) model, Ann. Appl. Probab. 14 (2) (2004) 971-1005.

[19] É. Le Page, Théorème de renouvellement pour les produits de matrices aléatoires et les équations aux différences aléatoires, in : Séminaires de probabilités Rennes 1983, Univ. Rennes I, Rennes, 1983, 116 pp.

[20] I.I. Piatetski-Shapiro, Geometry of Classical Domains and Theory of Automorphic Functions, Sovremennye Problemy Matematiki, Gosudarstv. Izdat. Fiz.-Mat. Lit., Moscow, 1961.

[21] A. Raugi, Fonctions harmoniques sur les groupes localment compact a base denomerable, Bull. Soc. Math. France Mém. 54 (1977) 5-118. 